to appear in ApJ, accepted April 182011

\title{
Cool White Dwarfs Found in the UKIRT Infrared Deep Sky Survey
}

\author{
S. K. Leggett ${ }^{1}$ \\ N. Lodieu ${ }^{2,3}$ \\ P.-E. Tremblay ${ }^{4}$ \\ P. Bergeron ${ }^{4}$ \\ and \\ A. Nitta ${ }^{1}$
}

\begin{abstract}
We present the results of a search for cool white dwarfs in the United Kingdom InfraRed Telescope (UKIRT) Infrared Deep Sky Survey (UKIDSS) Large Area Survey (LAS). The UKIDSS LAS photometry was paired with the Sloan Digital Sky Survey (SDSS) to identify cool hydrogen-rich white dwarf candidates by their neutral optical colors and blue near-infrared colors, as well as faint Reduced Proper Motion magnitudes. Optical spectroscopy was obtained at Gemini Observatory, and showed the majority of the candidates to be newly identified cool degenerates, with a small number of G- to K-type (sub)dwarf contaminants. Our initial search of $280 \mathrm{deg}^{2}$ of sky resulted in seven new white dwarfs with effective temperature $T_{\text {eff }} \approx 6000 \mathrm{~K}$. The current followup of $1400 \mathrm{deg}^{2}$ of sky has produced thirteen new white dwarfs. Model fits to the photometry show that seven of the newly identified white dwarfs have $4120 \mathrm{~K} \leq T_{\text {eff }} \leq 4480 \mathrm{~K}$, and cooling ages between $7.3 \mathrm{Gyr}$ and $8.7 \mathrm{Gyr}$; they have $40 \mathrm{kms}^{-1} \leq v_{\tan } \leq 85$
\end{abstract}

\footnotetext{
${ }^{1}$ Gemini Observatory, Northern Operations Center, 670 N. A'ohoku Place, Hilo, HI 96720, USA

${ }^{2}$ Instituto de Astrofísica de Canarias (IAC), C/ Vía Láctea s/n, E-38200 La Laguna, Tenerife, Spain

${ }^{3}$ Departamento de Astrofísica, Universidad de La Laguna (ULL), E-38205 La Laguna, Tenerife, Spain

${ }^{4}$ Département de Physique, Université de Montréal, C.P. 6128, Succursale Centre-Ville, Montréal, QC H3C 3J7, Canada
} 
$\mathrm{kms}^{-1}$ and are likely to be thick disk 10-11 Gyr-old objects. The other half of the sample has $4610 \mathrm{~K} \leq T_{\text {eff }} \leq 5260 \mathrm{~K}$, cooling ages between $4.3 \mathrm{Gyr}$ and $6.9 \mathrm{Gyr}$, and $60 \mathrm{kms}^{-1} \leq v_{\tan } \leq 100 \mathrm{kms}^{-1}$. These are either thin disk remnants with unusually high velocities, or lower-mass remnants of thick disk or halo late-F or G stars.

Subject headings: white dwarfs — techniques: photometric — techniques: spectroscopic — Infrared: Stars — surveys

\section{Introduction}

White dwarfs are the end stage of stellar evolution for the vast majority of stars - all stars with initial mass between around $0.07 M_{\odot}$ and $8 M_{\odot}$ end their lives as cooling white dwarfs. The cooling rate of these degenerate remnants slows as their temperature drops, such that very old white dwarfs are still visible. The coolest white dwarfs can constrain the age of the Galactic disk, or even of the halo (e.g. Winget et al. 1987). While other methods for dating the Galactic components exist - asteroseismology, gyrochronology, isochrone fitting, isotope decay, magnetic activity, kinematics and metallicity (e.g. Ulrich 1986, Barnes 2007, Chaboyer et al. 1996, Frebel et al. 2007, West et al. 2008, Nordstrom et al. 2004, see also the review Soderblom 2010) - very cool white dwarfs are unambiguously old and their cooling rates are quite well understood, enabling their use as accurate chronometers (e.g. Iben \& Tutukov 1984; Fontaine, Brassard \& Bergeron 2001). Most white dwarfs consist of a C/O core with an outer envelope composed of helium and/or hydrogen, with occasional traces of metals. The mass and composition of both the core and the atmosphere are important in determining the age of a white dwarf. The mass and core composition determine the thermal content, while the composition of the atmosphere provides the insulating material which controls the rate of cooling. Bergeron et al. (2001) use atmospheric and evolutionary models to analyse a sample of white dwarfs with measured trigonometric parallaxes to show that the coolest of these white dwarfs, with $T_{\text {eff }} \sim 4000-4500 \mathrm{~K}$, are 9-10 Gyr old if they have a thick hydrogen atmosphere, and 8-9 Gyr old if they have a helium-rich atmosphere. These ages are consistent with the age of the local Galactic disk (e.g. Leggett et al. 1998).

Several groups are trying to find old white dwarfs to confirm the age of the disk and to investigate the ages of the older Galaxy components. Candidate white dwarfs are typically identified as high proper motion objects, or by their colors or spectra, or by a combination of their kinematic and photometric properties. Using kinematic data alone is problematical. Oppenheimer et al. (2001) identified a sample of high-velocity white dwarfs which was inferred to be a halo population by their kinematics. However Reid et al. (2001) suggest 
that the majority of this sample has kinematics consistent with thick disk membership, and analysis of the sample by Bergeron et al. (2005) found that the white dwarfs were relatively warm, implying relatively short cooling ages. The short cooling age does not necessarily exclude the possibility that these white dwarfs are old - they may have evolved from low-mass stars with long main-sequence lifetimes.

Omitting infrared data in studies of candidate cool white dwarfs can also lead to large uncertainties in the derived $T_{\text {eff }}$ and hence age. This is because the complete optical to infrared spectral energy distribution is required to determine the atmospheric composition and $T_{\text {eff }}$. Hydrogen-rich white dwarfs cooler than about $5000 \mathrm{~K}$ (depending on instrument resolution) are featureless, however the infrared region demonstrates the presence of hydrogen due to the opacity of pressure-induced $\mathrm{H}_{2}$ absorption (e.g. Borysow 2002). Kilic et al. (2010a) perform a detailed analysis of cool white dwarfs using optical spectroscopy and infrared photometry. They determine significantly different values of $T_{\text {eff }}$ from those found using optical data only; several white dwarfs thought to have $T_{\text {eff }} \sim 3500 \mathrm{~K}$ in fact have $T_{\text {eff }} \sim 4500 \mathrm{~K}$, and the cooling age decreased from $>10$ Gyr to $<8$ Gyr.

Very cool white dwarfs are unambiguously old, as their total age is dominated by the large, post-main sequence, cooling time. There are about 20 white dwarfs known with $T_{\text {eff }}<4000 \mathrm{~K}$. These have been found as high proper-motion objects in photographic sky surveys (Hambly et al. 1997, Harris et al. 1999, Ibata et al. 2000, Oppenheimer et al. 2001, Rowell et al. 2008, Ruiz \& Bergeron 2001, Scholz et al. 2002) or in the Sloan Digital Sky Survey (SDSS, York et al. 2000) by inspection of spectra or by using the Reduced Proper Motion (RPM; Gates et al. 2004, Hall et al. 2008, Harris et al. 2001, Harris et al. 2008, Kilic et al. 2006, Kilic et al. 2010b). The RPM is defined as:

$$
H=\operatorname{mag}+(5 \times \log (P M)+5)
$$

where $P M$ is the proper motion in " $\mathrm{yr}^{-1}$, and the RPM acts as a proxy for absolute magnitude for a sample with similar kinematics. Faint white dwarfs with significant proper motion can be separated from stars and subdwarfs using an RPM diagram, such as that shown in Figure 1.

Our group has paired the UKIRT Infrared Deep Sky Survey (UKIDSS, Lawrence et al. 2007) with the SDSS, to provide a full optical through near-infrared spectral energy distribution, and to select candidate cool white dwarfs using the RPM and various colors. The proper motions are derived using the target coordinates and the epochs of the SDSS and UKIDSS images. Combining these databases allows us to go about a magnitude deeper in the $g$-band than other searches, to $g \approx 21$, although we are also limited to $r \approx 21$ by the time required to obtain a spectrum for classification. In Lodieu et al. (2009b) we published the results of a search of $280 \mathrm{deg}^{2}$ of the Large Area Survey (LAS) component of the UKIDSS 
Data Release (DR) 2. The search produced seven new white dwarfs with $T_{\text {eff }} \approx 6000 \mathrm{~K}$, which were confirmed with optical spectroscopy obtained at Gemini Observatory. Here we report the identification and confirmation of white dwarfs selected from $1400 \mathrm{deg}^{2}$ of DR 6 of the LAS. The larger area and better understanding of the photometry has allowed us to discover significantly cooler and older white dwarfs compared to the Lodieu et al. sample.

\section{Sample Selection}

\subsection{The LAS and SDSS Databases}

UKIDSS consists of five survey components, one of which is the LAS (Lawrence et al. 2007). The LAS is the sub-survey most likely to contain faint and rare sources of the local Galaxy, such as the cool white dwarfs and brown dwarfs. The LAS aims to survey 4000 $\operatorname{deg}^{2}$ in $Y J H K$ (Hewett et al. 2006) with a second epoch at $J$, to reach $J \sim 20$. The $5 \sigma$ photometric depths are $Y=20.2, J=19.6, H=18.8$ and $K=18.2$ (Dye et al. 2006). The data and catalogs generated by the automatic pipeline processing can be retrieved through the WFCAM Science Archive (WSA; Hambly et al. 2008). All data are pipeline-processed by the Cambridge Astronomical Survey Unit (CASU) following a standard procedure for infrared images (Irwin et al. 2004). An extensive description of each step involved in the processing of the WFCAM data is available on the CASU webpage 1 .

The work presented here involves spectroscopic follow-up during Gemini Observatory semesters 2008B and 2010A (see §3). Our selection of candidates evolved over time, as LAS Data Releases became available, and as we obtained spectra for the candidates. The Observatory was flexible in allowing us to replace targets listed in the proposals with better candidates as they were found in new Data Releases. The LAS Releases used were DR3 (December 2007, $809 \mathrm{deg}^{2}$ ), DR4 (July 2008, $984 \mathrm{deg}^{2}$ ), DR5 (April 2009, $1270 \mathrm{deg}^{2}$ ) and DR6 (October 2009, $1434 \mathrm{deg}^{2}$ ).

We have cross-correlated the LAS with the SDSS database, using Structured Query Language (SQL) and the WSA. SDSS Data Releases 6 and 7 were used, as available (AdelmanMcCarthy et al. 2008, Abazajian et al. 2009). The area surveyed by the LAS was designed to overlap with the SDSS, divided up into three blocks (Dye et al. 2006, Lawrence et al. 2007, Warren et al. 2007). The equatorial block with Right Ascension 22 to 04 hours and Declination between -1.5 and +1.5 degrees overlaps SDSS stripes 9 to 16 . The southern block covers 8 to 16 hours and (approximately) -3 to +15 degrees, and includes SDSS stripe

\footnotetext{
${ }^{1}$ http://casu.ast.cam.ac.uk/surveys-projects/wfcam/technical
} 
82. The northern block covers 8 to 17 hours and (approximately) +30 to +50 degrees, and provides an overlap with SDSS stripes 26 to 33.

\subsection{Astrometry and Photometry}

Sources were matched by requiring the presence of a "primary" SDSS source within 5 " of the LAS coordinates (increasing the search radius led to erroneous pairings). The WFCAM astrometry is tied to the 2MASS point source catalog (Skrutskie et al. 2006) and has a systematic accuracy of $<0$ '.1 rms (Dye et al. 2006). Lodieu et al. (2009) plot the $\mathrm{rms}$ of the difference between the coordinates of all point sources in the LAS DR2 and SDSS DR5 as a function of $J$ magnitude; for the sources in this sample with $17.5 \leq J \leq 19.2$ the implied astrometric uncertainty is $00^{\prime \prime} 025$ to $0{ }^{\prime \prime} 035$ for the brighter to fainter sources, respectively.

We have restricted our queries of the LAS database to detections classified as point sources, and imposed color criteria and lower limits on the $g$-band RPM, $H_{g}$. The queries return coordinates, photometry and errors from both surveys, as well as the proper motion, computed from the difference between the LAS J-band coordinates and the SDSS z-band coordinates. For the sample presented here, the SDSS epoch ranges from 1999 to 2006, and the LAS from 2005 to 2008. The average time period between the LAS and SDSS astrometry is $4.5 \pm 2.0$ years. Given the astrometric uncertainty, the uncertainty in proper motion is $\lesssim$ 14 mas. For one source, ULAS J1323+12, the LAS and SDSS epochs are separated by only $0.89 \mathrm{yr}$ and the proper motion uncertainty is 30 mas.

Table 1 gives astrometric information for the candidate white dwarfs studied here, and Table 2 gives the SDSS ugriz and the LAS YJHK photometry. Note that the photometry is taken from the 8th Data Releases for both the LAS and SDSS, and not the releases used for our initial source identification. The proper motion in Table 1 is similarly an updated value, and has been calculated from the coordinates and epochs given in the DR8 releases.

With the exception of the close binary ULAS J1234+06, Table 1 also lists proper motions derived from alternative catalog matching. For 10 of the 16 sources, proper motion derived by matching the USNO-B catalog (Monet et al. 2003) to the SDSS catalog is available via DR8 of the SDSS. Of these ten, there is good agreement for six objects. For two the differences are at the $\sim 2 \sigma$ level (ULAS J0121-00 and ULAS J1142+00). For the remaining two objects the discrepancies are large, and we rederived the proper motions using the digitised sky images, as well as the USNO-B, SDSS and LAS astrometry, in order to obtain the motion over a large timeline. For ULAS J1323+12, which has a small SDSS-LAS epoch difference, the derived motion is close to the USNO-SDSS value, and significantly smaller 
than the SDSS-LAS value. For ULAS J2246-00, the derived value is close to the SDSS-LAS value but significantly different from the USNO-SDSS value in direction. For the six objects without USNO-SDSS proper motions, three show good agreement between the SDSS-LAS proper motions and new measurements derived from the imaging and catalog data listed above. Three objects differ by $\sim 2 \sigma$ (ULAS J0840+05, ULAS J1345+15, ULAS J1454-01). Table 1 gives the RPM values derived from both proper motion measurement sets.

\subsection{Color and RPM Selection}

Figures 1 and 2 illustrate the selection criteria imposed to arrive at the candidate list of Table 1. For all queries, we selected $14 \leq J \leq 19.6$, to avoid saturated sources, and to ensure a good $(5 \sigma) J$-band detection, in the LAS. We also restricted the targets to $r_{A B}<20.7$, so that the spectroscopic observations were of reasonable duration (see $\S 3.1$ ). To select for faint high-proper motion sources, we restricted sources to $H_{g}>20.5$ (see Figure 1). This last

selection greatly reduced the number of objects returned from a query. Figure 3 shows the red to far-red colors of various samples, for completeness.

Most sources, 9 of the 17 listed in Table 1, were found in the search identified as region A in Figures 1 and 2, and in Table 1. The cuts imposed, in addition to those listed above, were: $H<18.9,0.8 \leq g-r \leq 1.6,0.2 \leq r-i \leq 0.6,0.6 \leq i-J \leq 1.4, J-H \leq 0.2$. This selection is designed to produce hydrogen-rich white dwarfs with $T_{\text {eff }} \sim 4000 \mathrm{~K}$ (Figure 2 ), with a good detection in the $H$-band. Early on in this work an additional source on the blue side of this cut with $g-r=0.63$ was followed up; later we prioritized redder (in $g-r$ ) sources in order to find white dwarfs cooler than $5000 \mathrm{~K}$. This bluer source is identified as found in region $\mathrm{Ab}$ in Table 1. An additional 6 sources were found in search $\mathrm{B}$, defined as: $0.2 \leq g-r \leq 1.2,-0.6 \leq r-i \leq+0.6, J-H \leq-0.1, H-K \leq-0.1$. This search is designed to find bluer sources where $\mathrm{H}_{2}$ opacity is impacting the red as well as the near-infrared, due to either lower $T_{\text {eff }}$, or a higher-pressure mixed H-He atmosphere (Figure 2). Finally, one more source was found by selecting for extreme sources in the RPM diagram (Figure 1): $H_{g}>22.5,-0.5 \leq g-i \leq 1.0,0.0 \leq r-i \leq 0.6, H<18.8, J-H \leq 0.1, H-K \leq 0$. This source is identified as found in region $\mathrm{C}$ in Table 1 (later the RPM value was found to be erroneous).

Our spectroscopic followup is complete for the pairing of the $1270 \mathrm{deg}^{2}$ of the LAS DR5 and the SDSS DR7 with the following selection: $H_{g}>20.5, r<20.7,14 \leq J \leq 19.6, H<$ $18.9,0.9 \leq g-r \leq 1.6,0.2 \leq r-i \leq 0.6,0.6 \leq i-J \leq 1.4, J-H+\left(\sqrt{\operatorname{err}(J)^{2}+\operatorname{err}(H)^{2}}\right)<$ 0.2. This search of the $1270 \mathrm{deg}^{2}$ of LAS DR5 produced six new white dwarfs with $4120 \mathrm{~K}$ $\leq T_{\text {eff }} \leq 4380 \mathrm{~K}$ (see $\S 4$ ), and recovered a known white dwarf with $T_{\text {eff }}=4390 \mathrm{~K}$ (SDSS 
J115814.52+000458.3, Kilic et al. 2010a). Kilic et al. (2010a) perform a detailed analysis of 113 white dwarfs with $v_{\tan } \geq 20 \mathrm{kms}^{-1}$ found using an RPM search of the $5282 \mathrm{deg}^{2}$ footprint of the SDSS DR3 (Harris et al. 2006). We can compare our results to those of Kilic et al., neglecting the additional 13 white dwarfs in the Kilic et al. sample that were found through a search of SDSS spectral data, in order to compare similarly determined samples. Kilic et al. find 6 white dwarfs with $4100 \mathrm{~K} \leq T_{\text {eff }} \leq 4400 \mathrm{~K}$ out to a distance of about $55 \mathrm{pc}$. The implied space density of $4200 \mathrm{~K}$ white dwarfs, with cooling age $\approx 8 \mathrm{Gyr}$ $(\S 4)$, is $2 \times 10^{-5} \mathrm{pc}^{-3}$. The $r_{-}, J$ - and $H$-band cuts imposed for our LAS search indicate that we should be sensitive to $4200 \mathrm{~K}$ white dwarfs at distances of $10-95$ pc, based on the models. The $H_{g}$ selection combined with a faint limit of $g \approx 21$ implies that our lower limit on proper-motion is $0^{\prime} \cdot 08 \mathrm{yr}^{-1}$. Our upper limit is determined by the $5^{\prime \prime}$ matching radius and the difference between the SDSS and LAS epochs; the typical difference is four years implying an upper limit to the proper motion of $\sim 1^{\prime \prime} .3 \mathrm{yr}^{-1}$. Excluding ULAS J1142+00 and ULAS J1234+06AB for which erroneously high proper motions were initially derived, the sample has a range in proper motion of $0^{\prime \prime} .09$ to $00^{\prime \prime} .3 \mathrm{yr}^{-1}$. The density of $4200 \mathrm{~K}$ white dwarfs implied by the Kilic et al. sample suggests that we would expect to find around seven $4200 \mathrm{~K}$ white dwarfs. However two of the six Kilic et al. white dwarfs have $H_{g}<20.5$, suggesting that our selection would find around five $4200 \mathrm{~K}$ white dwarfs, very close to the number found.

We note in passing that we have applied this last selection to the $48 \mathrm{deg}^{2}$ overlap area of the UKIDSS Galactic Cluster Survey (GCS) DR8 and SDSS DR7, and found no candidates. The GCS will survey ten open star clusters in the $Z Y J H K$ filters, to a similar depth in $Y J H K$ as the LAS. It has been successfully used to find field brown dwarfs (Lodieu et al. 2009a), as well as to study open clusters (Lodieu et al. 2007), but a larger area in common with the SDSS is required before cool white dwarfs will be found.

\section{Observations}

\subsection{Optical Spectroscopy}

We obtained optical spectra of the candidate cool white dwarfs listed in Table 1, selected as described in the previous Section, excluding SDSS J1247+06 for which Kilic et al. 2010a present an optical spectrum (a spectrum is also available in the SDSS database). The Gemini Multi-Object Spectrographs (GMOS, Hook et al. 2004) at both Gemini North and South were used, through queue time granted under programs GS-2008B-Q-35, GN-2008BQ-111 and GS-2010A-Q-58. For all observations the R400 grating was used with the GG455

blocking filter. The central wavelength was $680 \mathrm{~nm}$, with wavelength coverage of $460-890$ 
$\mathrm{nm}$. Detector fringing affected the spectra longwards of about $800 \mathrm{~nm}$. We did not correct for the fringing as this wavelength region is not necessary for target classification. The 0 '.75 slit was used with $2 \times 2$ binning, and the resulting resolution was $R \approx 1280$ or $6 \AA$. An observing log is given in Table 3 .

Flatfielding and wavelength calibration were achieved using lamps in the on-telescope calibration unit. Spectrophotometric DA white dwarf standards were used to determine the instrument response curve, and flux calibrate the spectra; for program GS-2008B-Q-35 LTT 7987 was used, for GN-2008B-Q-111 G191-B2B was used, and for GS-2010A-Q-58 LTT 3218 was used. The data were reduced using routines supplied in the IRAF Gemini package.

Figure 4 shows the GMOS spectra of the three non-white dwarf objects in our sample, as well as G- and K-type stellar spectra taken from the spectral atlas of Le Borgne et al. (2003) for reference, for a wavelength range that contains the features useful for classification. The narrow metal lines seen in the spectra exclude the possibility that these are white dwarfs. The three objects (ULAS J1142+00, ULAS J1234+06N and ULAS J2246-00) are early-G to early-K dwarfs or subdwarfs. For two of these, ULAS J1142+00 and ULAS J1234+06N, we initially derived too high a proper motion, the revised smaller $H_{g}$ value places them near the subdwarf region of the RPM (Figure 1). Our true contamination is therefore small, one in fifteen, or $7 \%$.

Figure 5 shows the spectra for the thirteen newly confirmed white dwarfs. Two of these show weak pressure-broadened H $\alpha$ (ULAS J1323+12 and ULAS J2331+15), and eleven are featureless.

\subsection{Infrared Photometry}

When carrying out our analysis by fitting models to the observed photometry ( $\$ 4$ ), we found several instances where the infrared photometry appeared to be in error, in particular for the $H$-band. To trace the source of the discrepancies, we obtained repeat photometry for a subset of the sample.

$H$ and $K$ photometry for five of our targets was obtained using the Wide Field Camera (WFCAM, Casali et al. 2007) on UKIRT, through the UKIRT Service program, via program USERV1876. Single-pointing WFCAM "paw-print" observations were defined to place the target in one of the four cameras: camera number 2. Exposure times of $10 \mathrm{~s}$ were used, with an 8 position telescope dither pattern, and a 4 position microstep pattern. These 32 exposures were repeated three times, for a total on-source time of 16 minutes, in each filter. An observing $\log$ is given in Table 4, together with the derived photometry. The 
"flat-file" FITS images and catalogs produced by the CASU pipeline were accessed through the WSA (§2). The $2^{\prime \prime}$-diameter aperture photometry, provided in counts, were converted to magnitudes using the airmass and zeropoints provided for each of the three reduced groups of observations for each filter and each object. We derived the weighted mean (using the uncertainties provided in the catalog) and the uncertainty in the mean for these measurements. Subsequently, the merged catalog data was released for the three objects observed on 2010 June 13, and the photometry from this catalog, database USERV1876v20101104, is given in the Table for ULAS J1206+03, ULAS J1351+12 and ULAS J1404+13. The merged catalog photometry agreed with the flatfile photometry to within 0.02 magnitudes for the two brighter sources, within $3 \%$ at $H$ for the fainter ULAS J1404+13, and within $12 \%$ at $K$ for ULAS J1404+13, which has $K \approx 19.7$. These differences are well within the quoted uncertainties.

Additional $H$, or $H$ and $K$, photometry was obtained for five more white dwarfs using NIRI (Hodapp et al. 2003) on Gemini North, via program GN-2011A-Q-59. Exposure times of $15 \mathrm{~s}$ or $30 \mathrm{~s}$ were used, with a 5 or 9 position telescope dither pattern. The total integration time is given in Table 4, together with the derived photometry. The data were reduced using routines supplied in the IRAF Gemini package. UKIRT Faint Standards were used for calibration (FS 16, FS 33, FS 126, FS 136; Leggett et al. 2006).

Comparing Table 4 to the LAS photometry in Table 2, shows that typically, for the sources fainter than $H \approx 18$, the LAS $H$-band magnitude is too faint by about $2 \sigma$. In selecting faint sources near the LAS detection limit, with blue $J-H$ colors, objects have been scattered into the sample with $H$ magnitudes too faint by twice the estimated uncertainty. A similar effect is seen in searches for faint brown dwarfs that are also blue in $J-H$ (B. Burningham, private communication, 2010).

\section{Analysis}

\subsection{Model Fitting}

The pure-hydrogen, pure-helium and mixed composition model atmospheres used in this analysis are described in detail in Kilic et al. (2010a). These models are in local thermodynamic equilibrium, they allow energy transport by convection, and they can be calculated with arbitrary amounts of hydrogen and helium. For the sample considered here, the mixed composition atmospheres had ratios of $\mathrm{He}$ to $\mathrm{H}$ by number ranging from low values of $10^{-2}$ to high values of $10^{10}$. The method used to fit the photometric data is described at length in Bergeron et al. (2001). Briefly, we convert the magnitudes into observed fluxes 
using the method of Holberg \& Bergeron (2006) and the appropriate filters. We use the SDSS to AB system corrections for $u, i$ and $z$ given in Eisenstein et al. (2006, Section 2). Then we fit the resulting energy distributions with those derived from model atmosphere calculations, using a nonlinear least-squares method. Only $T_{\text {eff }}$ and the solid angle $\pi(R / D)^{2}$, where $R$ is the radius of the star and $D$ is its distance from Earth, are considered free parameters. Since no parallax measurements are available, we assume a surface gravity of $\log g=8$ which determines the value of $R$ for a given value of $T_{\text {eff }}$ (Bergeron et al. 2001). White dwarfs have been shown to have a very strongly peaked mass and surface gravity distribution (e.g. Bergeron et al. 1992; Liebert, Bergeron \& Holberg 2005; Kepler et al. 2007). DA white dwarfs have a mean mass of $0.6 \pm 0.1 M_{\odot}$ while DBs are slightly more massive with $0.7 \pm 0.1 M_{\odot}$; these ranges infer a likely range in gravity for our sample of $7.7 \leq \log g \leq 8.3$. The photometric variance uncertainties in $T_{\text {eff }}$ and the solid angle are obtained directly from the covariance matrix of the fit.

Since our models do not include the red wing opacity from Ly $\alpha$ calculated by Kowalski \& Saumon (2006), we neglect here the $u$ bandpass in our fitting procedure as well as the $g$ bandpass for white dwarfs cooler than $4600 \mathrm{~K}$ in hydrogen-rich solutions (as the missing Ly $\alpha$ opacity has a larger impact at lower temperatures). The Ly $\alpha$ opacity affects a wavelength region where there is very little flux; hence the atmospheric structure is not affected significantly by a change of the opacity in the ultraviolet, although the predicted blue colors are.

As described in $§ 3.2$, we have found that in selecting faint sources near the LAS detection limit, with blue $J-H$ colors, objects have been scattered into the sample with $H$ magnitudes too faint by twice the estimated uncertainty. In our fits to the data we use the NIRI and WFCAM photometry where available (Table 4), and where LAS photometry is used we exclude data with uncertainties $\geq 0.15$ magnitudes. The fits use the DR 8 releases of both the SDSS and LAS photometry, the most recent at the time of writing.

We use the energy distributions together with the optical spectra at $\mathrm{H} \alpha$ to constrain the surface composition. Only two of the white dwarfs show $\mathrm{H} \alpha$ (Figure 5), however this does not necessarily imply that the remaining objects are helium-rich, as $\mathrm{H} \alpha$ absorption is not seen in hydrogen-rich white dwarfs cooler than $\sim 5000$ K. Figures 6 though 11 show the model fits to the observational data, assuming $\log g=8.0$.

The energy distributions are somewhat sensitive to $\log g$ in the temperature range considered here. A variation of \pm 0.3 dex in $\log g$ yields differences in effective temperature of between $40 \mathrm{~K}$ and $80 \mathrm{~K}$. The uncertainty due to the photometric variations is between $40 \mathrm{~K}$ and $150 \mathrm{~K}$. Another error estimate is provided by fitting the observed $\mathrm{H} \alpha$ line profile for the two DAs: the derived $T_{\text {eff }}$ differs from that derived from the energy distribution by 100 
- 200 K (for ULAS J1323+12 5400 K cf. 5260 K, for ULAS J2331+15 5200 K cf. 4970 K). This is consistent with our other error estimates.

\subsection{Derived Properties}

Table 5 lists the derived atmospheric properties of the 13 new white dwarfs in our sample, together with the SDSS white dwarf recovered here. Using the composition and temperature, and assuming that these stars have the canonical white dwarf mass of $0.6 M_{\odot}$, we can use the synthetic colors of Holberg \& Bergeron (2006, an extension of Bergeron, Wesemael \& Beauchamp 1995) and the evolutionary sequences of Fontaine, Brassard \& Bergeron (2001) to derive both a cooling age and distance, and hence tangential velocity. These values are also given in Table 5. The derived tangential velocities, $H_{g}$ and $g-i$ are consistent with the $40-150 \mathrm{kms}^{-1}$ region indicated in Figure 1. The uncertainty in the implied cooling age, distance and velocity are primarily due to the uncertainty in gravity (or mass). The sense of the gravity effect is that more massive white dwarfs will have a longer cooling age and be closer and slower, and vice versa.

Four of the thirteen newly identified white dwarfs $(\sim 30 \%)$ are best fit with purehydrogen atmospheres (Figures 6 and 7; ULAS J0826-00, ULAS J1323+12, ULAS J1345+15, ULAS J2331 +15$)$, and four $(\sim 30 \%)$ are best fit with pure-helium atmospheres (Figure 8; ULAS J1006+09, ULAS J1206+03, ULAS J1351+12, ULAS J1454-01). An additional white dwarf (ULAS J1320+08) is fit with either pure-helium or a helium-rich atmosphere, where the latter fit, with $\mathrm{H} / \mathrm{He}=10^{-4.4}$, is superior (Figure 9). Two sources (ULAS J0840+05, ULAS J1404+13) have mixed atmospheres with large flux deficits in the infared, due to pressure-induced $\mathrm{H}_{2}$ absorption (Figure 10). As described in Kilic et al. (2010a), there are generally two solutions to such objects. The $\mathrm{H}_{2}$ opacity peaks around $\mathrm{H} / \mathrm{He}=10^{-2}$ and there is usually a good solution both above and below this peak with slightly different temperatures. This is demonstrated by the fits shown in Figure 10, where ULAS J0840+05 is fit with $\mathrm{H} / \mathrm{He}=10^{-4.5}$ or $10^{-0.6}$, and ULAS J1404+13 is fit with $\mathrm{H} / \mathrm{He}=10^{-2.8}$ or $10^{-2.1}$. Thus three white dwarfs $(\sim 25 \%)$ have mixed atmospheres, and are similar to the mixed composition white dwarfs found by Kilic et al. (2010a, their Figure 14). There are seven white dwarfs $(\sim 55 \%)$ that have pure-helium or helium-dominated atmospheres. Finally, there are two white dwarfs ( $\sim 15 \%$ of the sample) for which we cannot constrain the atmospheric composition: ULAS J0121-00 and ULAS J1436+05 (Figure 11). ULAS J1436+05 is particularly puzzling - it appears to be much too bright at $z Y J$. A binary solution does not seem likely given the flux distribution, and the photometric uncertainties are small. 


\subsection{Discussion}

Typically, studies of samples of cool white dwarfs find a much larger fraction of purehydrogen atmospheres: 50-60\% (Bergeron et al. 2001, Kilic et al. 2010a). However Kilic et al. (2010a) find that $\sim 60 \%$ of their sample of white dwarfs (25 of 40) in the temperature range 4500-5000 $\mathrm{K}$ have pure-helium atmospheres while only $\sim 20 \%$ have pure-hydrogen, consistent with our findings. As Kilic et al. state, further work is required to understand if the observed overabundance of helium-rich atmosphere white dwarfs at this temperature range is real, or if $\mathrm{Ly} \alpha$ or $\mathrm{H}_{2}$ opacity uncertainties or photometric errors have biassed the results.

Seven white dwarfs, half of our sample, have $4120 \mathrm{~K} \leq T_{\text {eff }} \leq 4480 \mathrm{~K}$ and cooling ages between 7.3 Gyr and 8.7 Gyr. Their distances are 60-80 pc, and their tangential velocities are $40-85 \mathrm{kms}^{-1}$. The main-sequence lifetime of these remnants is likely to have been $\sim 2$ Gyr (e.g. Catalán et al. 2008), hence these white dwarfs are most likely to be thick disk 10-11 Gyr-old objects. Of these seven, we could not constrain the atmospheric composition for two, two have mixed atmospheres, two pure hydrogen and one pure helium. We note that there are no pure helium atmosphere white dwarfs cooler than $4400 \mathrm{~K}$ in the Kilic et

al. (2010a) sample. The uncertainty in the photometry would allow ULAS J1006+09 to be hydrogen-rich, in which case it is slightly cooler, with $T_{\text {eff }}=4230 \mathrm{~K}$, and older, with a cooling age of 8.4 Gyr.

Four of the white dwarfs have $4610 \mathrm{~K} \leq T_{\text {eff }} \leq 4810 \mathrm{~K}$ and cooling ages between $6.5 \mathrm{Gyr}$ and 6.9 Gyr. Their distances are 65-100 pc, and velocities are 60-95 $\mathrm{kms}^{-1}$. The remaining three white dwarfs have $4970 \mathrm{~K} \leq T_{\text {eff }} \leq 5260 \mathrm{~K}$ and cooling ages between $4.3 \mathrm{Gyr}$ and 5.8 Gyr. Their distances are 60-100 pc, and velocities are 70-100 $\mathrm{kms}^{-1}$. This half of our sample may consist of thin disk remnants with unusually high velocities (e.g. Bergeron 2003, Tetzlaff et al. 2011), or lower-mass remnants of thick disk or halo late-F or G stars.

\section{Conclusions}

Our search of the $1400 \mathrm{deg}^{2}$ of SDSS and UKIDSS LAS (Data Release 6) sky for white dwarfs cooler than $5000 \mathrm{~K}$ resulted in 17 candidates. One of these was a previously known white dwarf, and three proved to be subdwarf or dwarf G- to K-type stars, as determined by GMOS spectroscopy obtained at Gemini Observatory. Of the thirteen newly confirmed white dwarfs, two show $\mathrm{H} \alpha$, the remaining are featureless. The relatively high number of subdwarfs in the sample is due to errors in the initial proper motion derivation, in one case due to an error in epoch determination, and in another to the binary nature of the source. 
We fit the SDSS and LAS ugrizYJHK photometry using model atmospheres which can be calculated with arbitrary amounts of hydrogen and helium. As parallaxes are not known for these objects, we adopted the canonical white dwarf surface gravity of $\log g=8.0$, although we explore the impact of varying gravity. We have found that in searching for objects faint at $H$, objects are scattered into our sample where the photometry is in error by twice the estimated uncertainty. Repeat $H$ - and $K$-band photometry proved to be necessary to fit the white dwarf energy distributions, where $H, K>18$ magnitudes.

Seven of the newly identified white dwarfs have $4120 \mathrm{~K} \leq T_{\text {eff }} \leq 4480 \mathrm{~K}$, and cooling ages between 7.3 Gyr and 8.7 Gyr; these are likely to be thick disk 10-11 Gyr-old objects. The other half of the sample has $4610 \mathrm{~K} \leq T_{\text {eff }} \leq 5260 \mathrm{~K}$, and cooling age between 4.3 Gyr and 6.9 Gyr. These are either thin disk remnants with unusually high velocities (e.g. Bergeron 2003, Tetzlaff et al. 2011), or lower-mass remnants of thick disk or halo late-F or G stars. Our earlier search of $280 \mathrm{deg}^{2}$ of LAS sky (Lodieu et al. 2009) resulted in seven spectroscopically confirmed new white dwarfs, with effective temperature $5400 \mathrm{~K} \leq T_{\text {eff }} \leq$ $6600 \mathrm{~K}$ and cooling age 1.8-3.6 Gyr; these are a more extreme example of apparently young objects with high tangential velocities.

We have determined that pairing the SDSS and LAS databases and using proper motion and color selections of: $H_{g}>20.5, r<20.7,14 \leq J \leq 19.6, H<18.9,0.9 \leq g-r \leq 1.6$, $0.2 \leq r-i \leq 0.6,0.6 \leq i-J \leq 1.4, J-H+\left(\sqrt{\operatorname{err}(J)^{2}+\operatorname{err}(H)^{2}}\right) \leq 0.2$ produces a reasonably complete sample of $4200 \mathrm{~K}$ white dwarfs, which have cooling ages of around 8 Gyr. When complete, the LAS should provide a sample of about twenty cool, thick disk, white dwarfs, and possibly identify white dwarf remnants of the halo. The results presented here for various color selections will be a useful guide for identifying cool white dwarfs in ongoing near-infrared sky surveys such as UKIDSS and the Visible and Infrared Survey Telescope for Astronomy (VISTA; McPherson et al. 2006). This is particularly true for white dwarfs showing significant pressure-induced $\mathrm{H}_{2}$ opacity, due to either extremely low temperatures or higher-pressure mixed H-He atmospheres. The near-infrared surveys will be useful for studying the complex spectral evolution of white dwarfs, by providing the ratio of hydrogen- to helium-rich white dwarfs at different values of $T_{\text {eff }}$.

We are very grateful to the referee, M. Kilic, for a review that greatly improved the paper.

Some of the data reported here were obtained as part of the United Kingdom Infrared Telescope (UKIRT) Service Programme; UKIRT is operated by the Joint Astronomy Centre on behalf of the Science and Technology Facilities Council of the U.K.. This paper makes extensive use of the UKIRT Infrared Deep Sky Survey (UKIDSS); we are grateful to the 
WFCAM instrument team, the UKIRT staff, the UKIDSS team, the CASU data processing team, and the WSA group at Edinburgh, all of whom have contributed to the success of the survey.

This paper also makes use of Sloan Digital Sky Survey (SDSS) data. Funding for the SDSS and SDSS-II has been provided by the Alfred P. Sloan Foundation, the Participating Institutions, the National Science Foundation, the U.S. Department of Energy, the National Aeronautics and Space Administration, the Japanese Monbukagakusho, the Max Planck Society, and the Higher Education Funding Council for England. The SDSS Web Site is http://www.sdss.org/. The SDSS is managed by the Astrophysical Research Consortium for the Participating Institutions. The Participating Institutions are the American Museum of Natural History, Astrophysical Institute Potsdam, University of Basel, University of Cambridge, Case Western Reserve University, University of Chicago, Drexel University, Fermilab, the Institute for Advanced Study, the Japan Participation Group, Johns Hopkins University, the Joint Institute for Nuclear Astrophysics, the Kavli Institute for Particle Astrophysics and Cosmology, the Korean Scientist Group, the Chinese Academy of Sciences (LAMOST), Los Alamos National Laboratory, the Max-Planck-Institute for Astronomy (MPIA), the MaxPlanck-Institute for Astrophysics (MPA), New Mexico State University, Ohio State University, University of Pittsburgh, University of Portsmouth, Princeton University, the United States Naval Observatory, and the University of Washington.

The Digitized Sky Surveys were produced at the Space Telescope Science Institute under U.S. Government grant NAG W-2166. The images of these surveys are based on photographic data obtained using the Oschin Schmidt Telescope on Palomar Mountain and the UK Schmidt Telescope. This research has made use of the VizieR catalogue access tool, CDS, Strasbourg, France.

This work is based on observations obtained at the Gemini Observatory, which is operated by the Association of Universities for Research in Astronomy, Inc., under a cooperative agreement with the NSF on behalf of the Gemini partnership: the National Science Foundation (United States), the Science and Technology Facilities Council (United Kingdom), the National Research Council (Canada), CONICYT (Chile), the Australian Research Council (Australia), Ministério da Ciência e Tecnologia (Brazil) and Ministerio de Ciencia, Tecnología e Innovación Productiva (Argentina).

SKL and AN's research is supported by Gemini Observatory. NL was funded by the Ramón y Cajal fellowship number 08-303-01-02. This work is also supported in part by the NSERC Canada and by the Fund FQRNT (Québec). 


\section{REFERENCES}

Abazajian, K. N. et al. 2009, ApJS, 182, 543

Adelman-McCarthy, J. K. et al. 2008, ApJS, 175, 297

Barnes, S.A. 2007, ApJ, 669, 1167

Bergeron, P. 2003, ApJ, 586, 201

Bergeron, P., Leggett, S. K. \& Ruiz, M.-T. 2001, ApJS, 133, 413

Bergeron, P., Ruiz, M.-T, Hamuy, M., Leggett, S. K., Currie, M. J., Lajoie, C.-P. \& Dufour, P. 2005, ApJ, 625, 838

Bergeron, P., Saffer, R. A. \& Liebert, J., 1992, ApJ, 394, 228

Bergeron, P., Wesemael, F. \& Beauchamp, A. 1995, PASP, 107, 1047

Borysow, A. 2002, A\&A, 390, 779

Casali, M. et al. 2007, A\&A, 467, 777

Catalán, S., Isern, J., García-Berro, E. \& Ribas, I. 2008, MNRAS, 387, 1693

Chaboyer, B., Demarque, P., Kernan, P. J. \& Krauss, L. M. 1996, Science, 271, 957

Dye, S. et al. 2006, MNRAS, 372, 1227

Eisenstein, D. J. et al. 2006, ApJS, 167, 40

Fontaine, G., Brassard, P. \& Bergeron, P. 2001, PASP, 113, 409

Frebel, A., Christlieb, N., Norris, J. E., Thom, C., Beers, T. C. \& Rhee, J. 2007, ApJ, 660, L117

Gates, E. et al. 2004, ApJ, 612, L129

Hall, P. B., Kowalski, P. M., Harris, H. C., Awal, A., Leggett, S. K., Kilic, M., Anderson, S. F. \& Gates, E. 2008, arXiv:0804.1570

Hambly, N. C., Smartt, S. J. \& Hodgkin, S. T. 1997, ApJ, 489, L157

Hambly, N. C. et al. 2008, MNRAS, 384, 637 
Harris, H. C., Dahn, C. C., Vrba, F. J., Henden, A. A., Liebert, J., Schmidt, G. D. \& Reid, I. N. 1999, ApJ, 524, 1000

Harris, H. C.et al. 2001, ApJ, 549, L109

Harris, H. C. et al. 2008, ApJ, 679, 697

Hewett, P. C., Warren, S. J., Leggett, S. K. \& Hodgkin, S. T. 2006, MNRAS, 367, 454

Hodapp, K. W. et al. 2003, PASP, 115, 1388

Holberg, J. B. \& Bergeron, P. 2006, AJ, 132, 1221

Hook, I., Jørgensen, I., Allington-Smith, J. R., Davies, R. L., Metcalfe, N., Murowinski, R. G. \& Crampton, D. 2004, PASP, 116, 425

Ibata, R., Irwin, M., Bienayme, O., Schlz, R. \& Guibert, J. 2000, ApJ, 532, L41

Iben, I., Jr. \& Tutukov, A. V. 1984, ApJ, 282, 615

Irwin et al. 2004, Optimizing Scientific Return for Astronomy through Information Technologies, ed. Quinn, P. J \& Bridger, A. Proceedings of the SPIE, 5493, 411.

Kepler, S. O., Kleinman, S. J., Nitta, A., Koester, D., Castanheira, B. G., Giovannini, O., Costa, A. F. M. \& Althaus, L. 2007, MNRAS, 375, 1315

Kilic, M. et al. 2006, AJ, 131, 582

Kilic, M. et al. 2010a, ApJS, 190, 77

Kilic, M. et al. 2010b, ApJ, 715, L21

Kowalski, P. M. \& Saumon, D. 2006, ApJ, 651, L137

Lawrence, A. et al. 2007, MNRAS, 379, 1599

Le Borgne, J. -F. et al. 2003, A\&A, 402, 433

Leggett, S. K. et al. 2006, MNRAS, 373, 781

Leggett, S. K., Ruiz, M. T. \& Bergeron, P. 1998, ApJ, 497, 294

Liebert, J., Bergeron, P. \& Holberg, J. B. 2005, ApJS, 156, 47

Lodieu, N., Dobbie, P. D., Deacon, N. R., Hodgkin, S. T., Hambly, N. C. \& Jameson, R. F. 2007, MNRAS, 380, 712 
Lodieu, N., Burningham, B., Hambly, N. C. \& Pinfield, D. J. 2009a, MNRAS, 397, 258

Lodieu, N., Leggett, S. K., Bergeron, P. \& Nitta, A. 2009b, ApJ, 692, 1506

McPherson, A. M. et al. 2006, Ground-based and Airborne Telescopes, edited by Larry M. Stepp, Proc. of SPIE Vol. 6267, 7

Monet, D. G. et al. 2003, AJ, 125, 984

Nordstrom, B. et al. 2004, A\&A, 418, 989

Oppenheimer, B. R., Hambly, N. C., Digby, A. P., Hodgkin, S. T. \& Saumon, D. 2001, Science, 292, 698

Reid, I. N., Sahu, K. C.; Hawley, S. L. 2001, ApJ, 559, 942

Rowell, N. R., Kilic, M. \& Hambly, N. C. 2008, MNRAS, 385, L23

Ruiz, M.-T. \& Bergeron, P. 2001, ApJ, 558, 761

Scholz, R.-D., Szokoly, G. P., Andersen, M., Ibata, R. \& Irwin, M. J. 2002, ApJ, 565, 539

Skrutskie, M. F. et al. 2006, AJ, 131, 1163

Soderblom, D. R. 2010, ARA\&A, 48, 581

Tetzlaff N., Neuhäuser, R. \& Hohle, M. M. 2011, MNRAS, 410, 190

Tokunaga, A. T. \& Vacca, W. D. 2005, PASP, 117, 421

Ulrich, R. K. 1986, ApJ, 306, L37

Warren, S. J. et al. 2007a, MNRAS, 375, 213

West, A. A., Hawley, S. L., Bochanski, J. J., Covey, K. R., Reid, I. N., Dhital, S., Hilton, E. J. \& Masuda, M. 2008, AJ, 135, 785

Winget, D. E., Hansen, C. J., Liebert, J., van Horn, H. M., Fontaine, G., Nather, R. E., Kepler, S. O. \& Lamb, D. Q. 1987, ApJ, 315, L77

York, D. G. et al. 2000, AJ, 120, 1579 


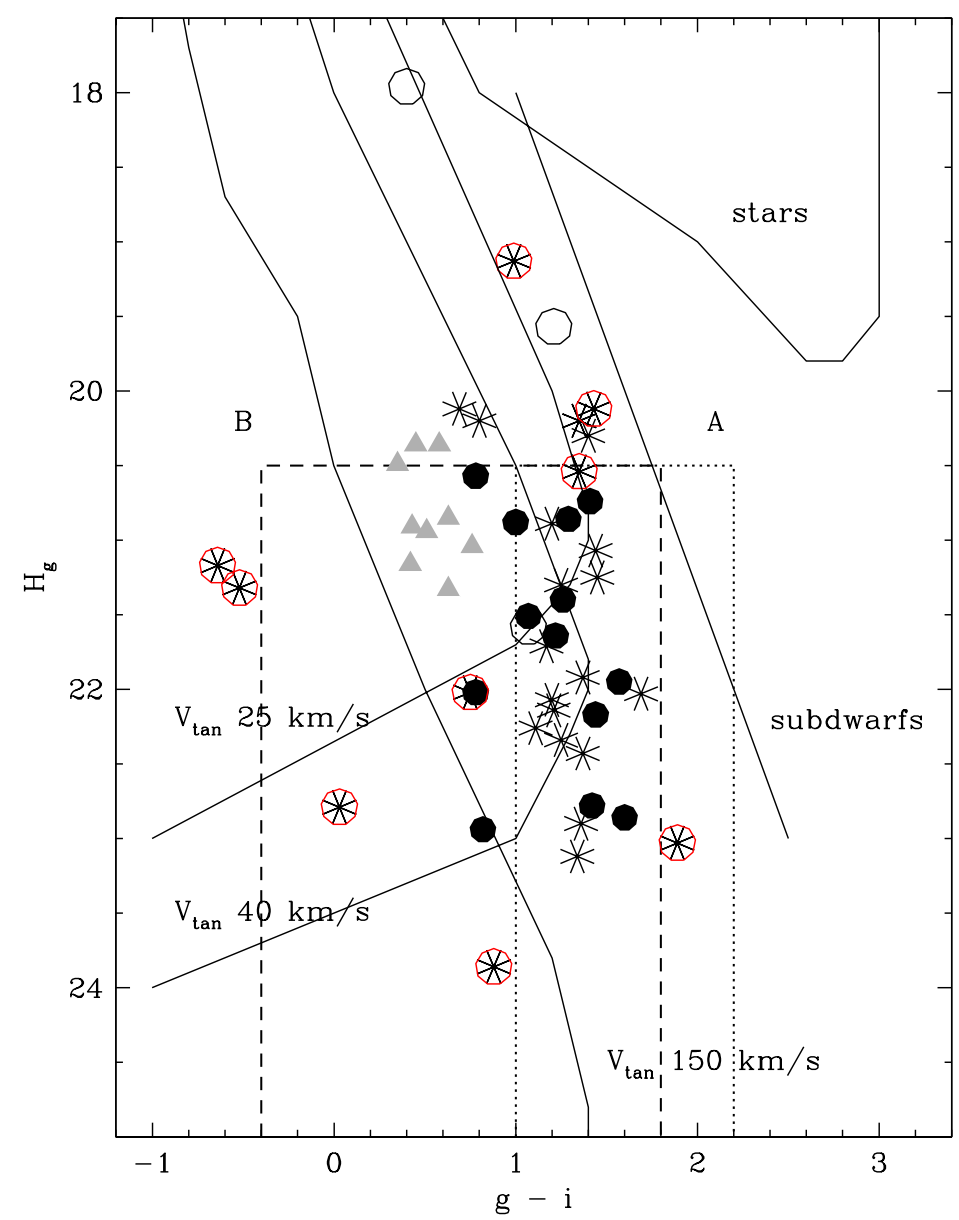

Fig. 1. - Reduced $g$-band proper motion as a function of $g-i$. White dwarf cooling curves for different tangential velocities are shown; the $v_{\text {tan }}=25-40 \mathrm{kms}^{-1}$ curves represent the expected location of disk white dwarfs, and the $v_{\tan }=150 \mathrm{kms}^{-1}$ curve the halo white dwarfs. The typical location of subdwarfs and stars are also shown (based on Figure 6 of Kilic et al. 2010a). Filled circles are confirmed white dwarfs and open circles are G- to Ktype (sub)dwarfs identified in this work. Grey triangles are the warmer sample of confirmed white dwarfs from Lodieu et al. (2009b). Asterisks are cool white dwarfs from Kilic et al. (2010a; white dwarfs identified both by the RPM method and spectroscopically by Gates et al. (2004) and Harris et al. (2008) are shown). Points circled in red are white dwarfs cooler than $4000 \mathrm{~K}$. The regions labelled A and B indicate the search criteria used here, see Figure 2 and the text. The photometry is taken from DR8 of the SDSS, and the proper motions are derived from the DR8 releases of the SDSS and LAS. 

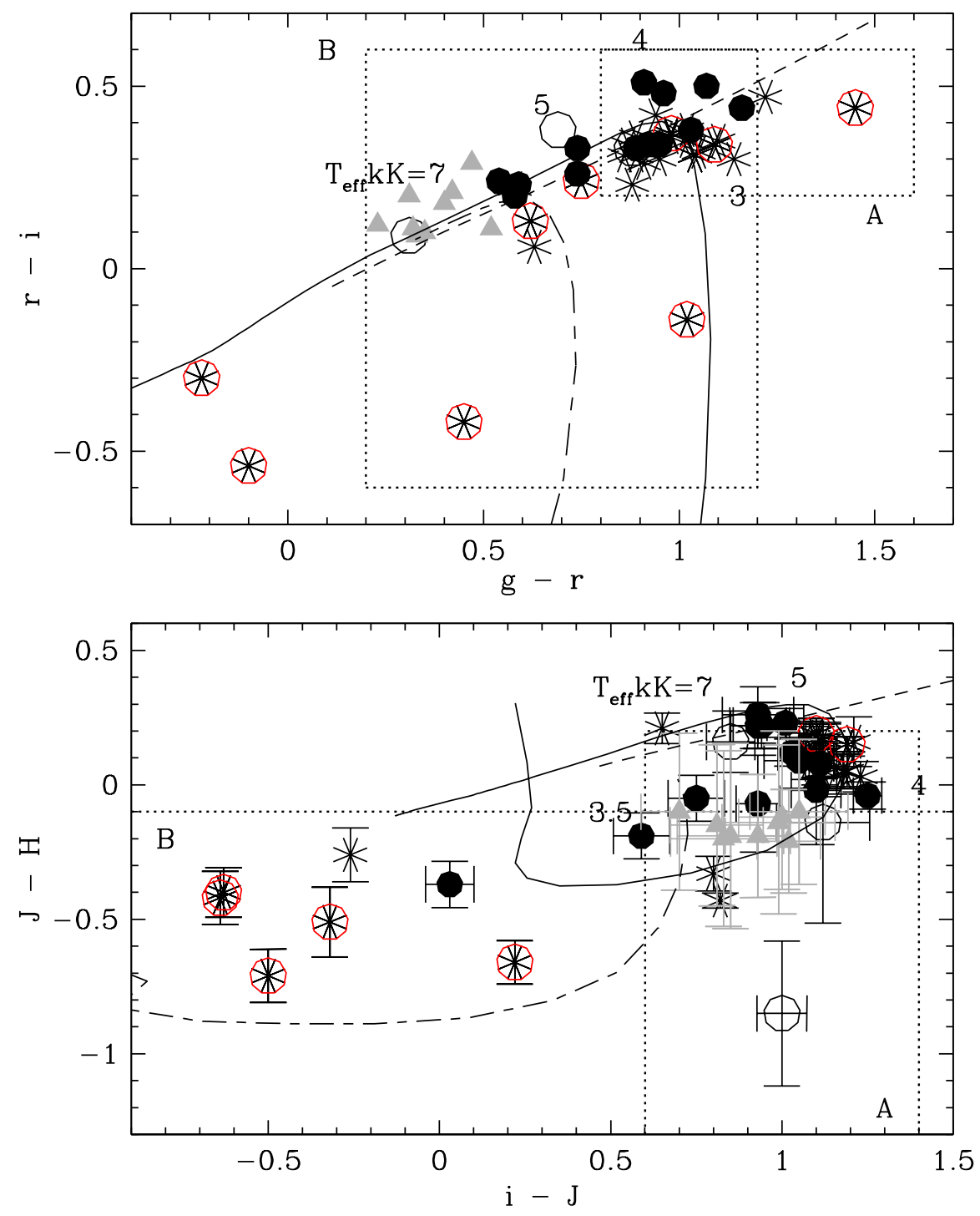

Fig. 2.- Plots demonstrating the color selection of the white dwarf candidates. Model sequences for white dwarfs with pure hydrogen and pure helium atmospheres are shown as solid and dashed lines, and a sequence with $\mathrm{He} / \mathrm{H}=100$ is shown as a short-long-dash line. $T_{\text {eff }} / 1000 \mathrm{~K}$ is indicated along the sequences for the pure hydrogen case. Symbols are as in Figure 1. The regions labelled A and B indicate the search criteria used here, as described in the text. The optical photometry is taken from SDSS DR8, and the infrared from our WFCAM and NIRI observations where available, otherwise from DR8 of the LAS. 

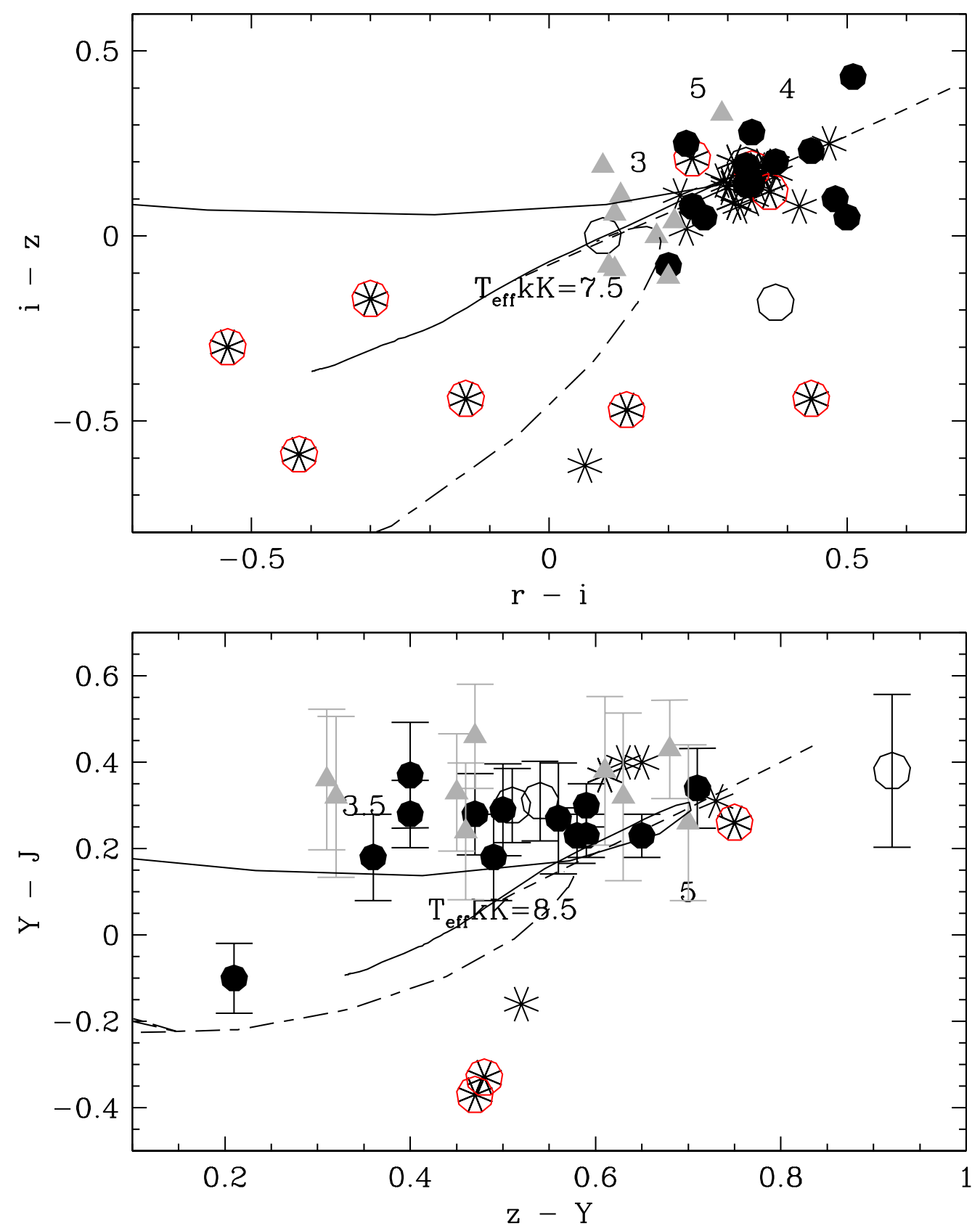

Fig. 3.- Plots of the red to far-red colors of the samples considered here. Symbols, sequences and data sources are as in Figures 2 and 3. 


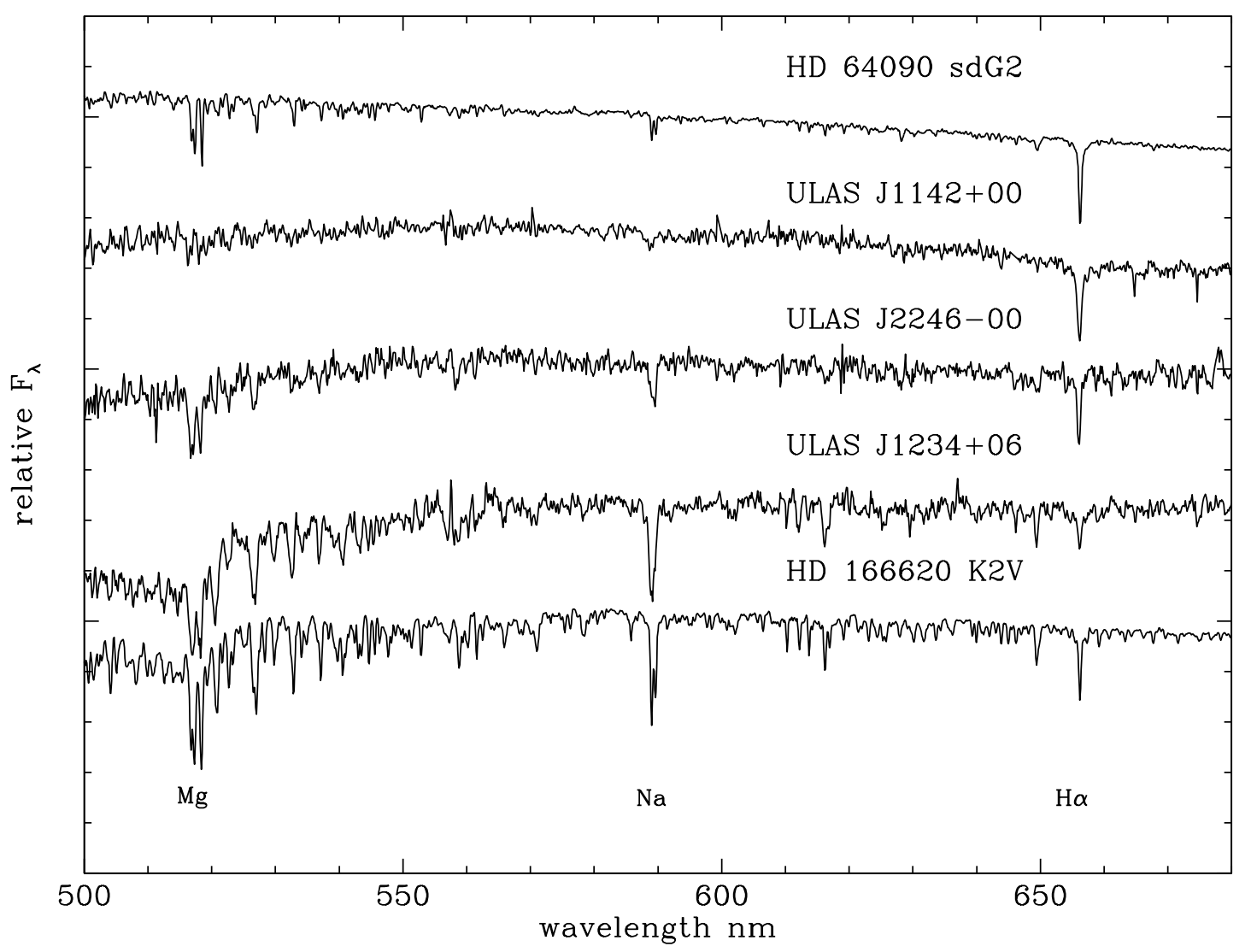

Fig. 4.- GMOS spectra of the three ULAS G and K stars in our sample, compared to similar spectra taken from Le Borgne et al. (2003; smoothed to the resolution of our data). The spectra have been normalized to unity at $600 \mathrm{~nm}$, and offset by 0.5 flux units for clarity. 


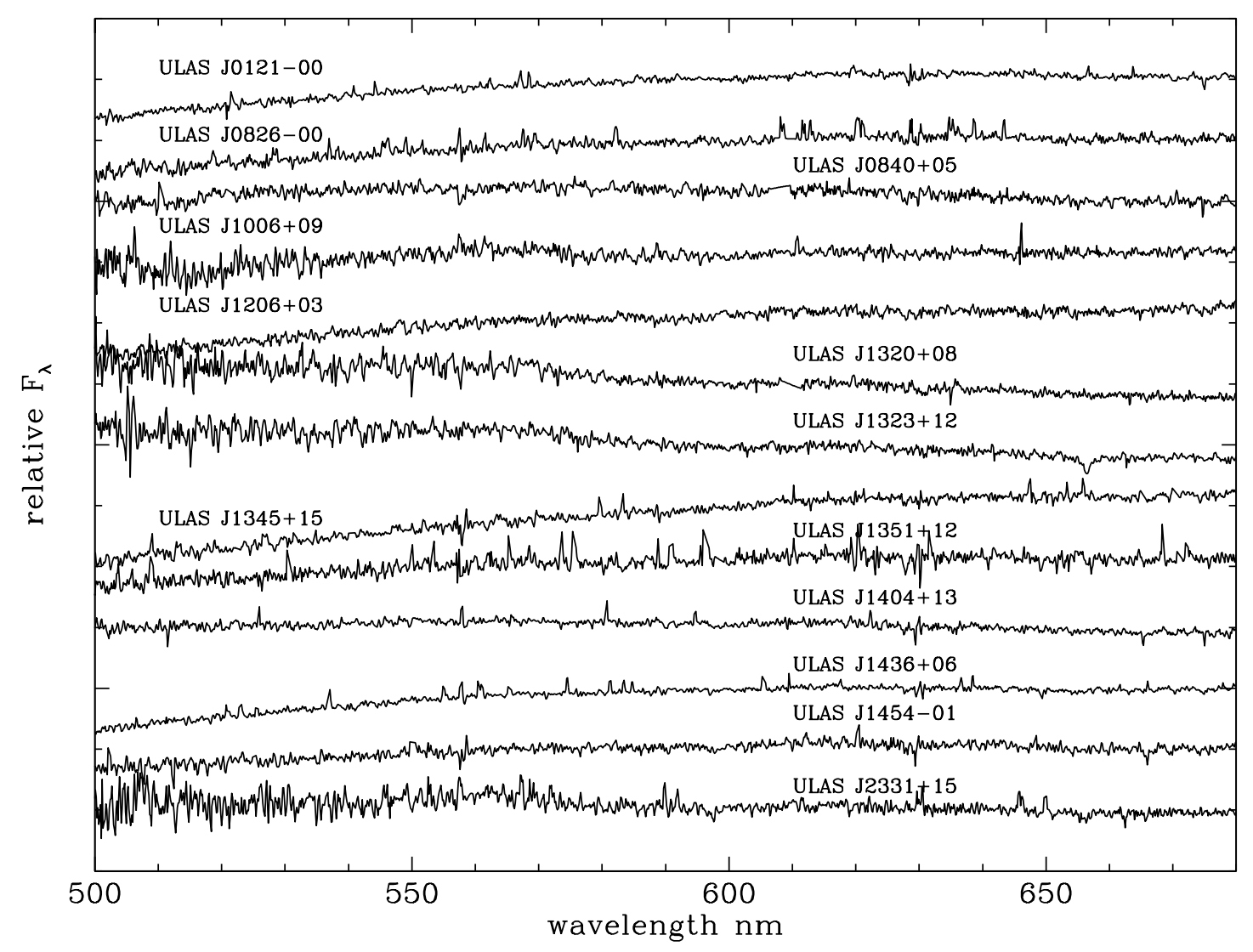

Fig. 5.- GMOS spectra of previously unknown white dwarfs in our sample. The spectra have been normalized to unity at $600 \mathrm{~nm}$, and offset by 0.5 flux units for clarity. $\mathrm{H} \alpha$ absorption is seen in the ULAS J1323+12 spectrum, and weakly in the ULAS J2331+15 spectrum (see also Figures 6 and 7). 

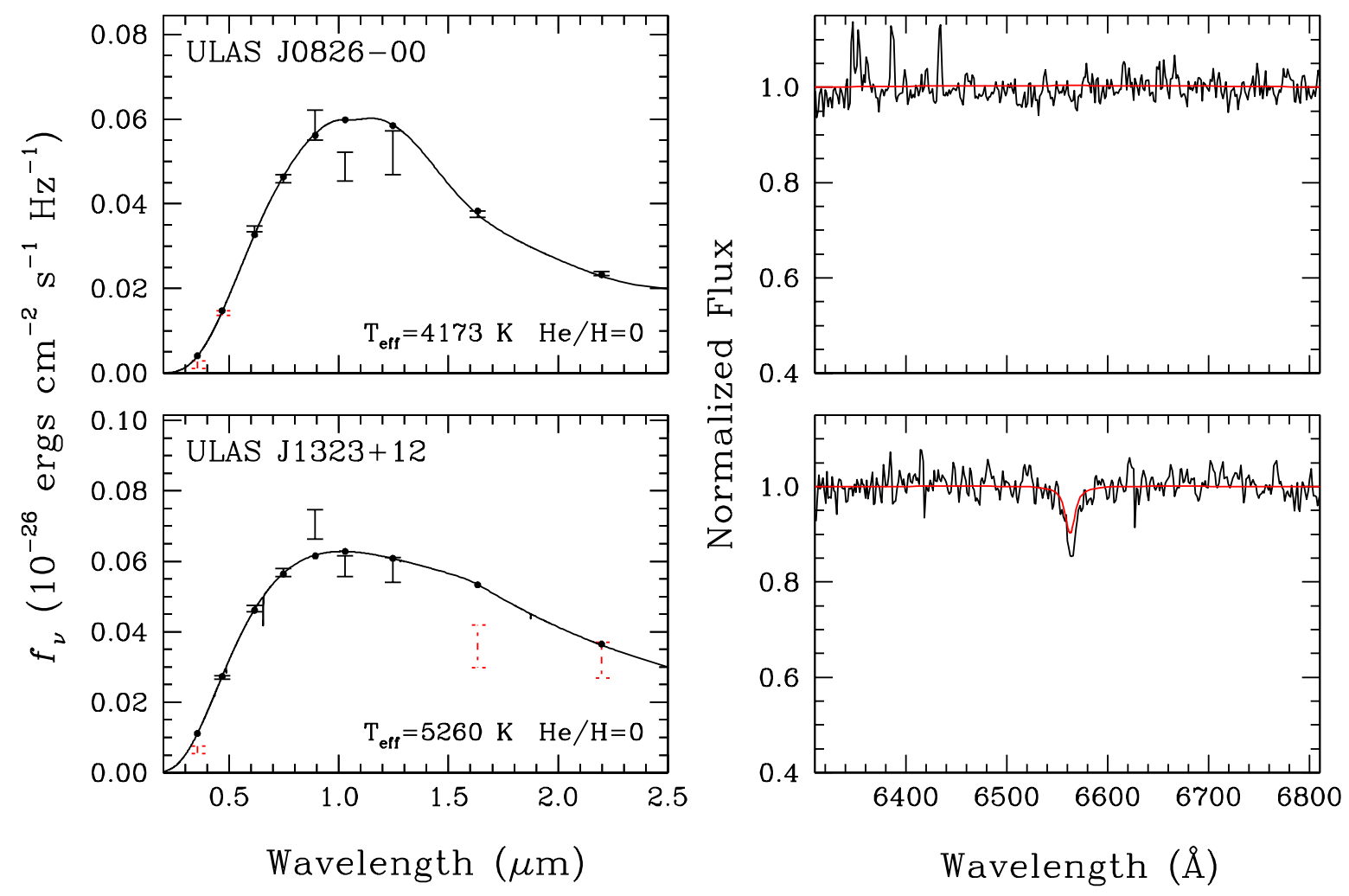

Fig. 6. - Two of the four white dwarfs in our sample best fit with pure hydrogen atmospheres. The error bars in the left panels represent SDSS ugriz and NIRI, WFCAM and/or LAS $Y J H K$ photometry. The dashed error bars indicate photometric datapoints that have been ignored in the fits: $u$ data, as well as $g$ for ULAS J0826-00, and $H$ and $K$ for ULAS $\mathrm{J} 1323+12$ (see text). The solid lines in the left panels represent the monochromatic model fluxes while the solid dots correspond to the average over the filter bandpass. A surface gravity $\log g=8.0$ is assumed, and the derived $T_{\text {eff }}$ is shown in the legends. The right panels show the observed spectrum around $\mathrm{H} \alpha$, with the modelled pure-hydrogen atmosphere line profiles. ULAS J0826-00 is too cool to show $\mathrm{H} \alpha$. 

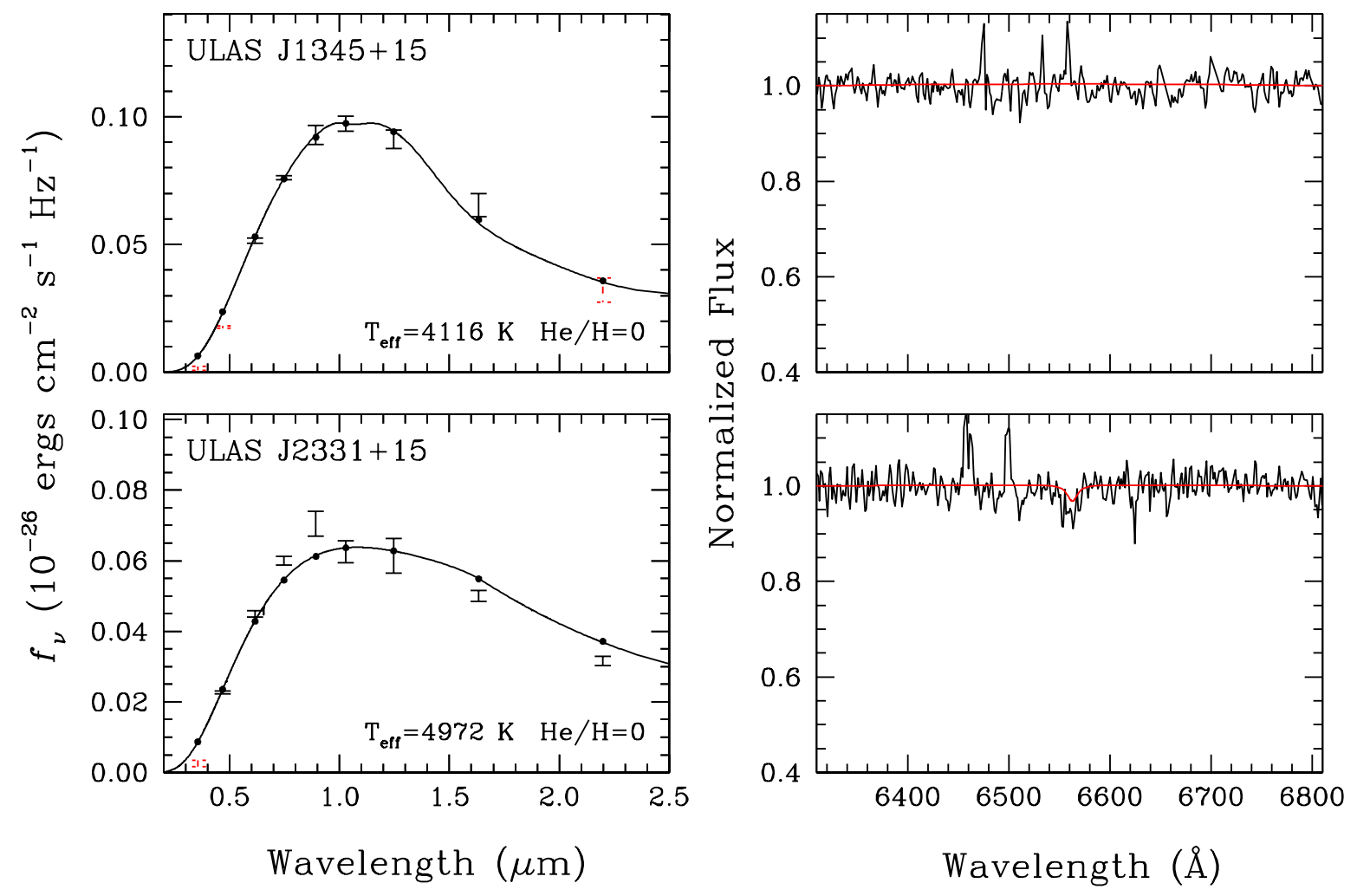

Fig. 7.- The two other white dwarfs in our sample best fit with pure hydrogen atmospheres. Symbols are as in Figure 6. $u$ data have been ignored in the fits, as well as $g$ and $K$ for ULAS J1345+15 (see text). ULAS J1345+15 is too cool to show H $\alpha$ (right panels). 


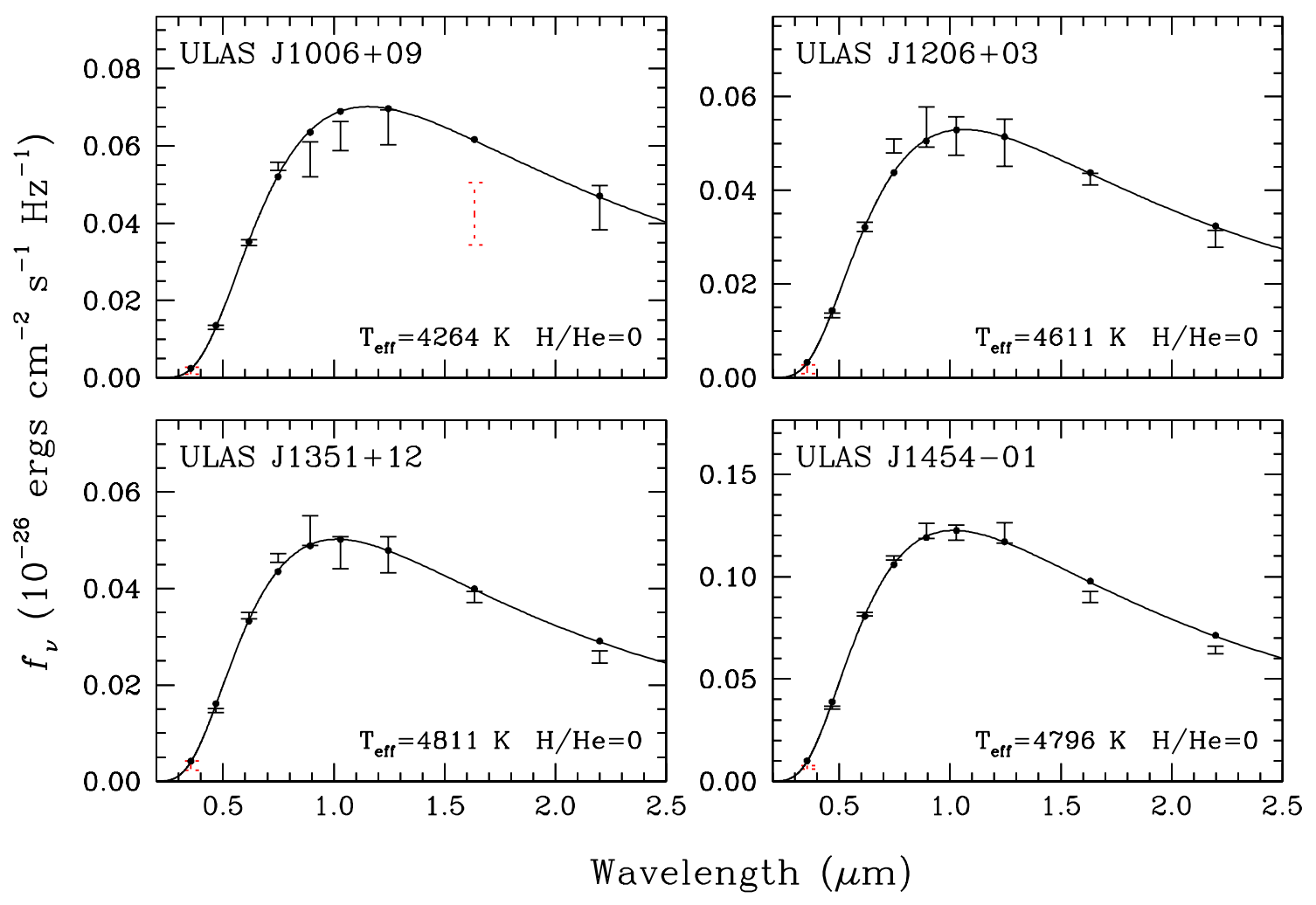

Fig. 8.- Four white dwarfs in our sample best fit with pure helium atmospheres. Symbols are as in Figure 6. $u$ data have been ignored in the fits, as well as $H$ for ULAS J1006+09 (see text). All objects have observed and modelled featureless spectra (not shown). 


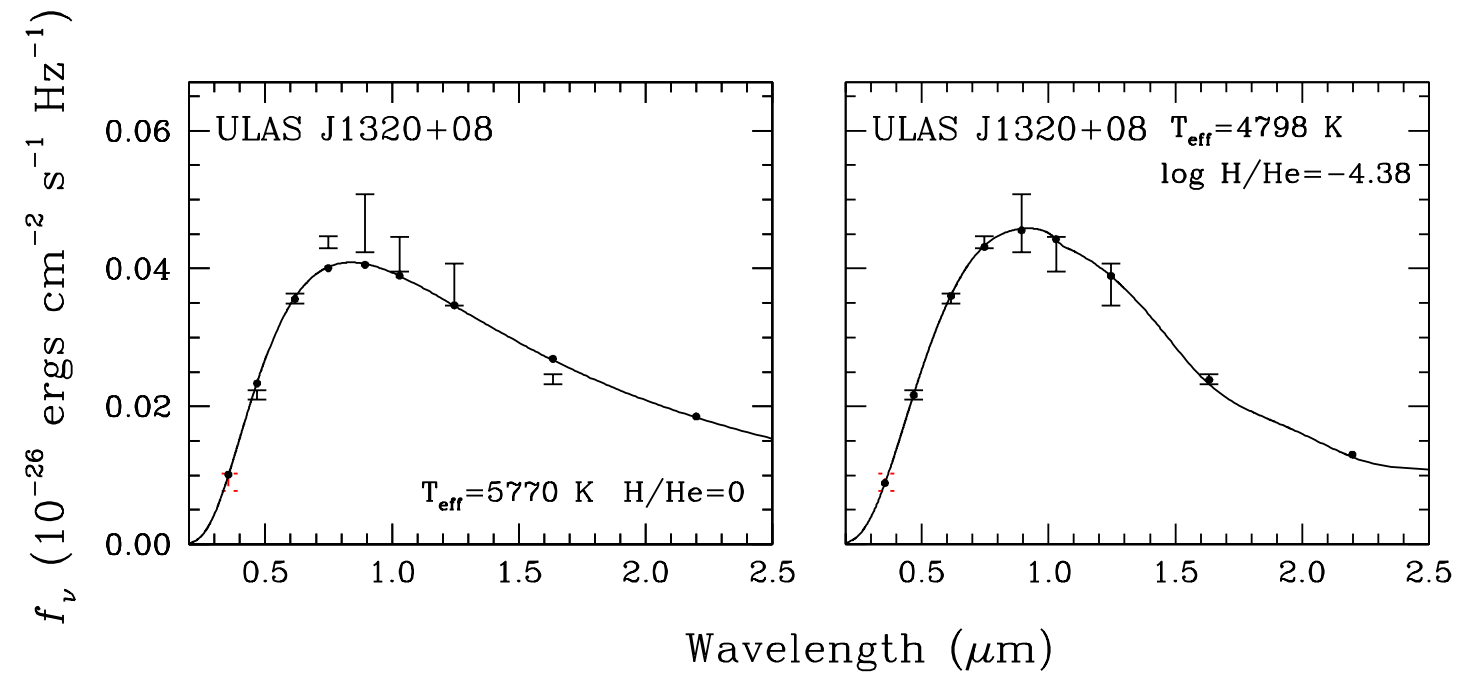

Fig. 9.- One white dwarf in our sample best fit with either a pure helium atmosphere (left panel) or a mixed atmosphere with a high $\mathrm{He} / \mathrm{H}$ ratio (right panel); the mixed fit is preferred. Symbols are as in Figure 6. $u$ data have been ignored in the fits (see text). Both the observed and modelled spectrum (not shown) is featureless. 


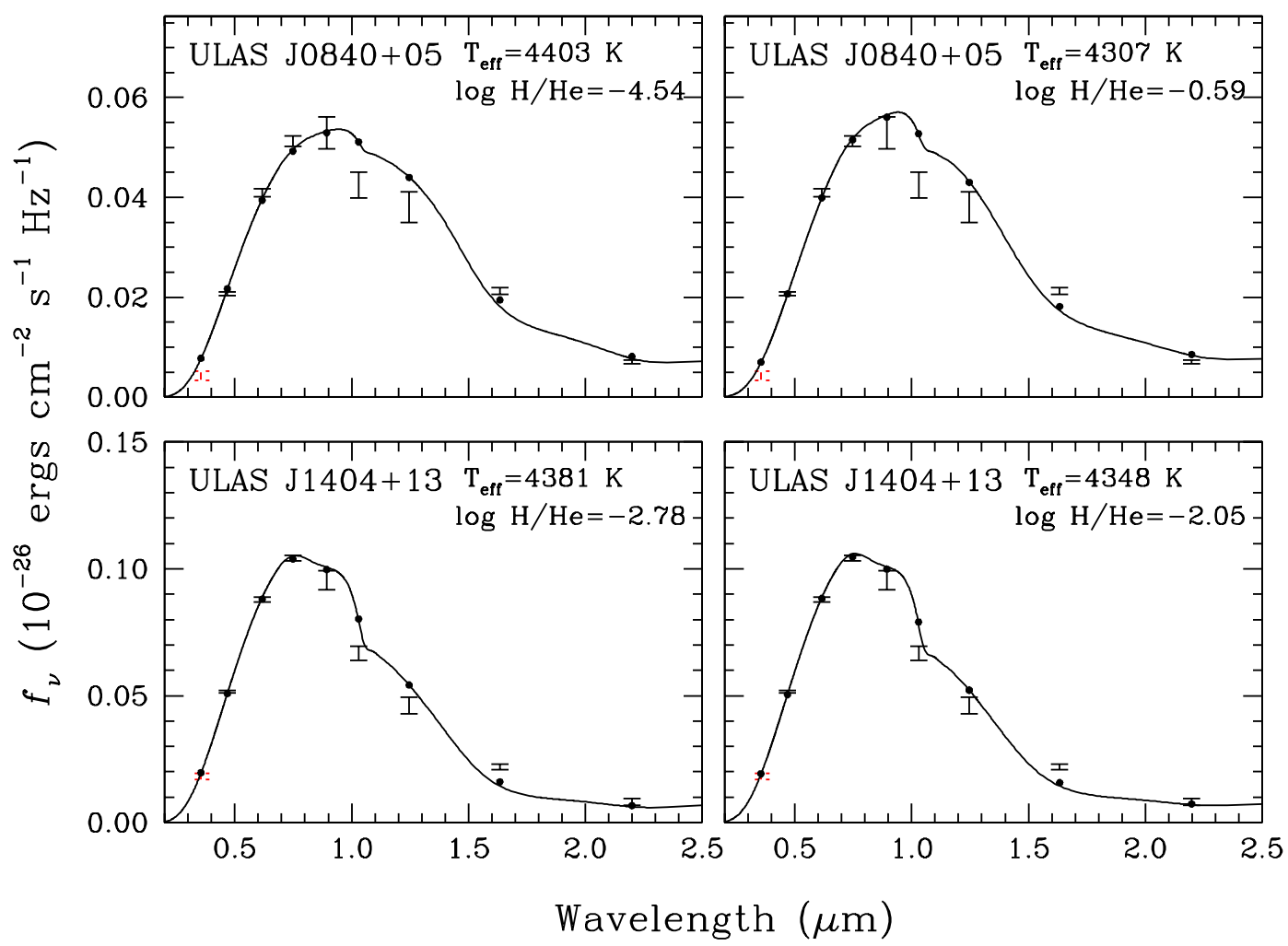

Fig. 10.- Two white dwarfs in our sample best fit with mixed $\mathrm{H} / \mathrm{He}$ atmospheres. Symbols are as in Figure 6. $u$ data have been ignored in the fits (see text). Solutions with two values of the $\mathrm{He} / \mathrm{H}$ ratio are possible (see text). Both objects have observed and modelled featureless spectra (not shown). 


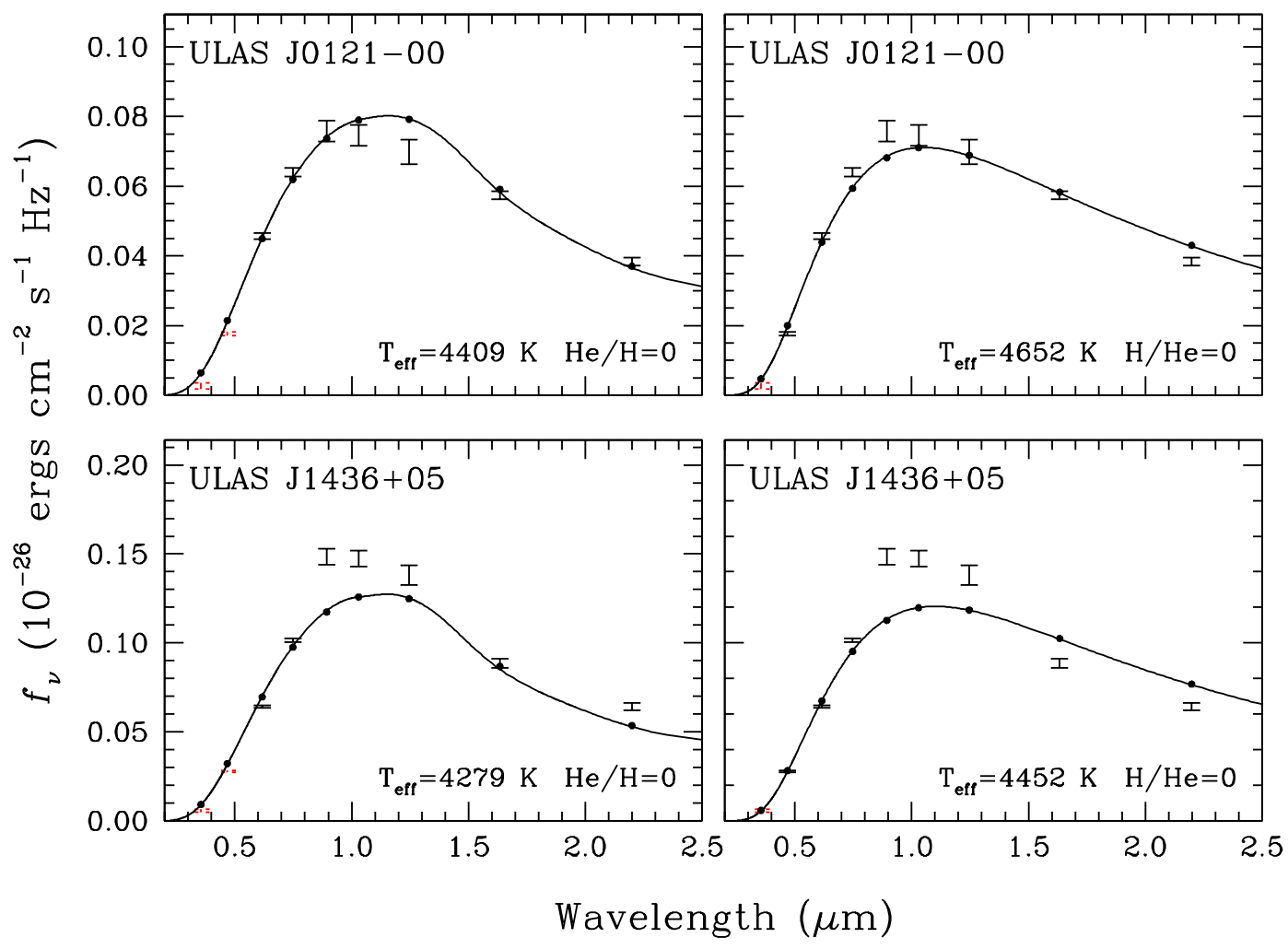

Fig. 11. - Two white dwarfs in our sample for which composition could not be constrained, the pure hydrogen fit is shown in the left panels, and the pure helium fit in the right panels. Symbols are as in Figure 6. $u$ data have been ignored in the fits, as well as $g$ in the pure hydrogen fits. Both objects have observed and modelled featureless spectra (not shown). 
Table 1. Astrometry for Candidate White Dwarfs

\begin{tabular}{|c|c|c|c|c|c|c|c|c|c|c|}
\hline \multirow[t]{2}{*}{ Short Name } & \multirow{2}{*}{$\begin{array}{l}\text { Right Ascension } \\
\text { HH:MM:SS.SS }\end{array}$} & \multirow{2}{*}{$\begin{array}{c}\text { Declination } \\
\text { DD:MM:SS.S }\end{array}$} & \multirow{2}{*}{$\begin{array}{c}\text { Epoch } \\
\text { YYYYMMDD }\end{array}$} & \multicolumn{2}{|c|}{ SDSS-LAS $\mu^{\prime \prime} \mathrm{yr}^{-1 \mathrm{a}}$} & \multicolumn{2}{|c|}{ Other $\mu^{\prime \prime} \mathrm{yr}^{-1 \mathrm{~b}}$} & \multirow{2}{*}{$\begin{array}{c}\mu^{\mathrm{b}} \\
\text { Note }\end{array}$} & \multirow{2}{*}{$\begin{array}{c}\mathrm{RPM}^{\mathrm{c}} \\
H_{g}\end{array}$} & \multirow{2}{*}{$\begin{array}{l}\text { Search } \\
\text { Region }\end{array}$} \\
\hline & & & & RA & Dec & $\mathrm{RA}$ & Dec & & & \\
\hline ULAS J0121-00 & 01:21:03.02 & $-00: 38: 33.4$ & 20061221 & +0.068 & +0.071 & +0.125 & +0.044 & 1 & 20.74 [21.39] & $\mathrm{A}$ \\
\hline ULAS J0826-00 & $08: 26: 44.57$ & $-00: 35: 47.6$ & 20061205 & +0.087 & +0.032 & +0.126 & +0.033 & 2 & 20.86 [21.59] & $\mathrm{A}$ \\
\hline ULAS J0840+05 & 08:40:01.43 & $+05: 15: 28.8$ & 20061216 & -0.030 & -0.109 & -0.102 & -0.076 & 2 & 20.88 [21.13] & $\mathrm{B}$ \\
\hline ULAS J1006+09e & 10:06:05.14 & $+09: 50: 15.2$ & 20070421 & +0.131 & -0.067 & +0.134 & -0.053 & 1 & $21.95[21.90]$ & $\mathrm{A}$ \\
\hline ULAS J1142+00 & $11: 42: 48.61$ & $+00: 12: 26.0$ & 20080415 & +0.050 & +0.011 & -0.007 & -0.007 & 1 & $17.96[14.39]^{\mathrm{g}}$ & $\mathrm{C}$ \\
\hline ULAS J1206+03 & $12: 06: 05.93$ & $+03: 47: 17.1$ & 20070405 & +0.021 & -0.163 & -0.008 & -0.160 & 2 & $22.17[22.11]$ & $\mathrm{B}$ \\
\hline ULAS J1234+06N & $12: 34: 40.82$ & $+06: 47: 19.8$ & 20070121 & +0.048 & -0.018 & & & & $19.57^{\mathrm{h}}$ & $\mathrm{B}$ \\
\hline ULAS J1234+06S ${ }^{\mathrm{i}}$ & $12: 34: 40.79$ & $+06: 47: 18.6$ & 20070121 & +0.072 & -0.001 & & & & $21.72^{\mathrm{h}}$ & \\
\hline SDSS J1247+06 j & $12: 47: 38.95$ & $+06: 46: 04.8$ & 20070121 & -0.383 & +0.072 & -0.385 & +0.071 & 1 & 22.98 [22.99] & $\mathrm{A}$ \\
\hline ULAS J1320+08 e & $13: 20: 16.50$ & $+08: 36: 43.6$ & 20070220 & -0.039 & -0.192 & +0.019 & -0.208 & 1 & 22.02 [21.62] & $\mathrm{B}$ \\
\hline ULAS J1323+12 & $13: 23: 57.27$ & $+12: 03: 13.4$ & 20070417 & +0.303 & -0.140 & +0.120 & -0.171 & 3 & $22.94[21.49]$ & $\mathrm{Ab}$ \\
\hline ULAS J1345+15 e & $13: 45: 50.68$ & $+15: 14: 54.5$ & 20080601 & -0.139 & -0.221 & -0.076 & -0.246 & 3 & $22.86[22.83]$ & $\mathrm{A}$ \\
\hline ULAS J1351+12 e & 13:51:09.60 & $+12: 14: 08.5$ & 20070421 & +0.024 & -0.119 & +0.002 & -0.104 & 1 & $21.40[21.07]$ & $\mathrm{A}$ \\
\hline ULAS J1404+13 e & 14:04:51.86 & $+13: 30: 55.6$ & 20080311 & -0.106 & +0.113 & -0.099 & +0.106 & 1 & $20.57[20.43]$ & $\mathrm{B}$ \\
\hline ULAS J1436+05 & $14: 36: 02.52$ & $+05: 38: 01.0$ & 20080526 & -0.092 & -0.301 & -0.102 & -0.284 & 2 & 22.78 [22.69] & $\mathrm{A}$ \\
\hline ULAS J1454-01 ${ }^{\mathrm{e}}$ & $14: 54: 27.16$ & $-01: 10: 05.8$ & 20080417 & -0.209 & -0.035 & -0.263 & -0.059 & 2 & $21.64[22.16]$ & $\mathrm{A}$ \\
\hline ULAS J2246-00f & $22: 46: 29.89$ & $-00: 50: 53.1$ & 20050906 & -0.111 & -0.009 & -0.097 & +0.012 & 3 & 21.58 [21.30] & $\mathrm{B}$ \\
\hline ULAS J $2331+15^{\mathrm{e}}$ & 23:31:39.91 & $+15: 18: 00.4$ & 20070930 & +0.136 & -0.082 & +0.113 & -0.097 & 1 & $21.51[21.37]$ & $\mathrm{A}$ \\
\hline
\end{tabular}

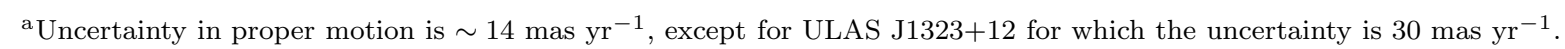

b Other sources: (1) SDSS-USNO proper motion reported in DR8 of the SDSS; (2) derived here from digital sky images together with the SDSS and LAS astrometry; (3) derived here from digital sky images, as well as the USNO-B, SDSS and LAS astrometry. Typical uncertainty is $\sim 10$ mas yr ${ }^{-1}$.

${ }^{\mathrm{c}}$ Value in brackets is that implied by the alternative proper motion value.

${ }^{\mathrm{d}}$ All region selections include $r_{A B}<20.7$ and $14 \leq J \leq 19.6$; in addition for region A $H_{g}>20.5, H<18.9,0.8 \leq g-r \leq 1.6,0.2 \leq r-i \leq 0.6$, $0.6 \leq i-J \leq 1.4, J-H \leq 0.2$. Region Ab is the same, except that the source is slightly bluer: $g-r=0.63$. Region B is defined by $H_{g}>20.5,0.2 \leq g-r \leq 1.2$, $-0.6 \leq r-i \leq+0.6, J-H \leq-0.1, H-K \leq-0.1$. Region $\mathrm{C}$ is defined by $H_{g}>22.5,-0.5 \leq g-i \leq 1.0,0.0 \leq r-i \leq 0.6, H<18.8, J-H \leq 0.1, H-K \leq 0$.

${ }^{\mathrm{e}}$ Confirmed as a white dwarf spectroscopically in this work.

${ }^{\mathrm{f}}$ Confirmed as a G- to K-type (sub)dwarf star spectroscopically in this work.

${ }^{g}$ Erroneously high initial proper motion determination placed object in sample. 
${ }^{\mathrm{h}}$ This close pair was incorrectly matched, resulting in a high initial proper motion determination which placed the Northern object in sample.

i No optical spectrum obtained.

${ }^{j}$ Previously discovered in SDSS by Kilic et al. (2010a). 
Table 2. SDSS DR8 and UKIDSS LAS DR8 Photometry for Candidate White Dwarfs

\begin{tabular}{|c|c|c|c|c|c|c|c|c|c|}
\hline Short Name & $u($ err $)$ & $g($ err $)$ & $r(\mathrm{err})$ & $i(\mathrm{err})$ & $z(\mathrm{err})$ & $Y($ err $)$ & $J($ err $)$ & $H($ err $)$ & $K($ err $)$ \\
\hline ULAS J0121-00 ${ }^{\mathrm{a}, b}$ & $22.87(0.30)$ & $20.78(0.03)$ & $19.75(0.02)$ & $19.37(0.02)$ & $19.17(0.04)$ & $18.59(0.04)$ & $18.36(0.05)$ & $18.56(0.18)$ & $18.06(0.23)$ \\
\hline ULAS J0826-00 ${ }^{\mathrm{a}, b}$ & $23.19(0.44)$ & $21.02(0.04)$ & $20.07(0.02)$ & $19.73(0.02)$ & $19.45(0.06)$ & $19.05(0.07)$ & $18.68(0.10)$ & $18.75(0.17)$ & \\
\hline ULAS J0840+05 ${ }^{\mathrm{a}, b}$ & $22.37(0.21)$ & $20.61(0.02)$ & $19.87(0.02)$ & $19.61(0.02)$ & $19.56(0.06)$ & $19.20(0.06)$ & $19.02(0.08)$ & $19.21(0.18)$ & \\
\hline ULAS J1006+09a & $23.26(0.48)$ & $21.11(0.04)$ & $20.04(0.02)$ & $19.54(0.02)$ & $19.49(0.08)$ & $18.78(0.06)$ & $18.44(0.07)$ & $18.46(0.19)$ & $17.90(0.13)$ \\
\hline ULAS J1142+00 & $20.47(0.04)$ & $19.41(0.01)$ & $19.10(0.01)$ & $19.01(0.01)$ & $19.01(0.03)$ & $18.47(0.06)$ & $18.16(0.07)$ & $18.00(0.09)$ & $17.95(0.15)$ \\
\hline ULAS J1206+03 ${ }^{\mathrm{a}, b}$ & $23.30(0.52)$ & $21.09(0.04)$ & $20.13(0.03)$ & $19.65(0.03)$ & $19.55(0.08)$ & $18.99(0.08)$ & $18.72(0.10)$ & $18.98(0.22)$ & $18.46(0.22)$ \\
\hline ULAS J1234+06 $\mathrm{N}^{\mathrm{c}}$ & $22.74(0.31)$ & $21.02(0.03)$ & $20.14(0.02)$ & $19.81(0.02)$ & $19.62(0.06)$ & $19.11(0.05)$ & $18.81(0.07)$ & $19.66(0.26)$ & $17.95(0.13)$ \\
\hline ULAS J1234+06S & $25.37(0.65)$ & $22.43(0.10)$ & $21.13(0.05)$ & $20.57(0.04)$ & $22.27(0.44)$ & $19.61(0.08)$ & $19.08(0.09)$ & $19.28(0.18)$ & \\
\hline SDSS J1247+06 ${ }^{\mathrm{e}}$ & $20.95(0.08)$ & $20.03(0.02)$ & $18.68(0.01)$ & $18.39(0.01)$ & $18.27(0.02)$ & $17.76(0.02)$ & $17.54(0.03)$ & $17.49(0.04)$ & $17.54(0.10)$ \\
\hline ULAS J1320+08 ${ }^{\mathrm{a}, b}$ & $21.56(0.14)$ & $20.56(0.03)$ & $20.02(0.02)$ & $19.78(0.02)$ & $19.70(0.09)$ & $19.21(0.06)$ & $19.03(0.08)$ & $19.53(0.16)$ & \\
\hline ULAS J1323+12 a & $21.91(0.17)$ & $20.32(0.02)$ & $19.73(0.02)$ & $19.50(0.02)$ & $19.25(0.06)$ & $18.85(0.05)$ & $18.57(0.06)$ & $18.64(0.17)$ & $18.25(0.16)$ \\
\hline ULAS J1345+15 a & $23.52(0.50)$ & $20.78(0.03)$ & $19.61(0.02)$ & $19.18(0.01)$ & $18.95(0.04)$ & $18.30(0.03)$ & $18.07(0.04)$ & $17.99(0.07)$ & $18.24(0.15)$ \\
\hline ULAS J1351+12 $2^{\mathrm{a}, b}$ & $22.66(0.29)$ & $20.98(0.03)$ & $20.06(0.02)$ & $19.72(0.02)$ & $19.58(0.06)$ & $19.08(0.07)$ & $18.79(0.08)$ & $18.88(0.16)$ & $18.63(0.19)$ \\
\hline ULAS J1404+13 ${ }^{\mathrm{a}, b}$ & $20.79(0.07)$ & $19.62(0.01)$ & $19.04(0.01)$ & $18.84(0.01)$ & $18.92(0.04)$ & $18.71(0.04)$ & $18.81(0.07)$ & $19.01(0.15)$ & \\
\hline ULAS J1436+05 & $22.05(0.17)$ & $20.29(0.02)$ & $19.38(0.01)$ & $18.87(0.01)$ & $18.44(0.03)$ & $17.85(0.03)$ & $17.62(0.04)$ & $17.51(0.06)$ & $17.47(0.11)$ \\
\hline ULAS J1454-01 ${ }^{\mathrm{a}, b}$ & $21.86(0.12)$ & $20.01(0.02)$ & $19.12(0.01)$ & $18.79(0.01)$ & $18.65(0.03)$ & $18.06(0.03)$ & $17.76(0.04)$ & $17.68(0.09)$ & $17.56(0.12)$ \\
\hline ULAS J2246-00 & $23.28(0.53)$ & $21.35(0.05)$ & $20.66(0.03)$ & $20.28(0.04)$ & $20.46(0.16)$ & $19.54(0.12)$ & $19.16(0.13)$ & $19.30(0.35)$ & \\
\hline ULAS J2331+15 & $22.92(0.33)$ & $20.51(0.02)$ & $19.77(0.02)$ & $19.44(0.02)$ & $19.25(0.05)$ & $18.78(0.05)$ & $18.50(0.08)$ & $18.72(0.25)$ & \\
\hline
\end{tabular}

Note. - SDSS ugriz magnitudes are on the AB system (Fukugita et al. 1996). LAS Y JHK are on the Vega system (Hewett et al. 2006).

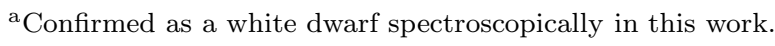

${ }^{\mathrm{b}}$ Improved $H$ and $K$ photometry is given in Table 4 .

${ }^{\mathrm{c}}$ Confirmed as a G- to K-type (sub)dwarf star spectroscopically in this work.

dNo spectrum obtained.

ePreviously discovered in SDSS by Kilic et al. (2010a). 
Table 3. GMOS Observation Log

\begin{tabular}{ccccl}
\hline \hline Short Name & $\begin{array}{c}\text { SDSS } r \\
\text { AB }\end{array}$ & $\begin{array}{c}\text { Total Exp. } \\
\text { seconds }\end{array}$ & $\begin{array}{c}\text { Date } \\
\text { YYYYMMDD }\end{array}$ & Program \\
\hline ULAS J0121-00 & & & & \\
ULAS J0826-00 & 19.70 & 5100 & 20100710 & GS-2010A-Q-58 \\
ULAS J0840+05 & 20.07 & 8400 & 20100119 & GS-2010A-Q-58 \\
ULAS J1006+09 & 19.83 & 3600 & 20081209 & GS-2008B-Q-35 \\
ULAS J1142+00 & 20.04 & 6400 & 20090122 & GN-2008B-Q-111 \\
ULAS J1206+03 & 19.11 & 1800 & 20100119 & GS-2010A-Q-58 \\
ULAS J1234+06N $\mathrm{N}^{\mathrm{b}, c}$ & 20.13 & 5040 & 20090120 & GS-2008B-Q-35 \\
ULAS J1320+08 & 19.76 & 5760 & 20090122 & GS-2008B-Q-35 \\
ULAS J1323+12 & 20.02 & 6400 & 20090126,20090127 & GN-2008B-Q-111 \\
ULAS J1345+15 & 19.77 & 4800 & 20090122,20090125 & GN-2008B-Q-111 \\
ULAS J1351+12 & 19.61 & 4400 & 20100415 & GS-2010A-Q-58 \\
ULAS J1404+13 & 20.07 & 8400 & 20100418,20100607 & GS-2010A-Q-58 \\
ULAS J1436+05 & 19.04 & 1800 & 20100508 & GS-2010A-Q-58 \\
ULAS J1454-01 & 19.40 & 3200 & 20100607,20100713 & GS-2010A-Q-58 \\
ULAS J2246-00 & 19.12 & 1800 & 20100418 & GN-2008B-Q-111 \\
ULAS J2331+15 & 20.64 & 11400 & 20080731 & GS-2008B-Q-35 \\
\hline
\end{tabular}

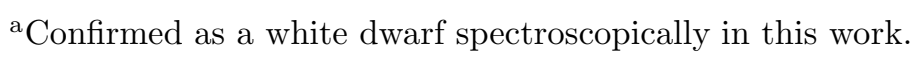

${ }^{\mathrm{b}}$ Confirmed as a G- to K-type (sub)dwarf star spectroscopically in this work.

${ }^{\mathrm{c}}$ Close pair of objects, spectroscopy is for northern source. 
Table 4. WFCAM and NIRI Photometry for LAS White Dwarfs

\begin{tabular}{cccccl}
\hline \hline Short Name & $H(\mathrm{err})$ & $K(\mathrm{err})$ & $\begin{array}{c}\text { Exp. } H, K \\
\text { minutes }\end{array}$ & $\begin{array}{c}\text { Date } \\
\text { YYYYMMDD }\end{array}$ & Program \\
& & & & & \\
\hline ULAS J0121-00 & $18.13(0.02)$ & $18.05(0.03)$ & 16,16 & 20100814,20100815 & USERV 1876 \\
ULAS J0826-00 & $18.59(0.02)$ & $18.58(0.02)$ & 9,9 & 20110126 & GN-2011A-Q-59 \\
ULAS J0840+05 & $19.21(0.03)$ & $19.89(0.06)$ & $13.5,18$ & 20110125 & GN-2011A-Q-59 \\
ULAS J1206+03 & $18.46(0.03)$ & $18.33(0.06)$ & 16,16 & 20100613 & USERV 1876 \\
ULAS J1320+08 & $19.08(0.03)$ & & 22.5 & 20110126 & GN-2011A-Q-59 \\
ULAS J1351+12 & $18.57(0.03)$ & $18.48(0.05)$ & 16,16 & 20100613 & USERV 1876 \\
ULAS J1404+13 & $19.18(0.05)$ & $19.72(0.16)$ & 16,16 & 20100613 & USERV 1876 \\
ULAS J1436+05 & $17.66(0.03)$ & $17.49(0.03)$ & $1.25,1.25$ & 20110105 & GN-2011A-Q-59 \\
ULAS J1454-01 & $17.64(0.03)$ & $17.49(0.03)$ & $1.25,1.25$ & 20110209 & GN-2011A-Q-59 \\
ULAS J2331+15 & $18.28(0.03)$ & $18.26(0.04)$ & 16,16 & 20100807,20100812 & USERV 1876 \\
\hline
\end{tabular}

Note. - HK are on the Vega system (Tokunaga \& Vacca 2005, Hewett et al. 2006). 
Table 5. Derived Properties of the LAS White Dwarfs

\begin{tabular}{ccccrrr}
\hline \hline Short Name & $\begin{array}{c}\text { Spectral } \\
\text { Type }\end{array}$ & $\begin{array}{c}\text { Atmospheric } \\
\text { Composition }\end{array}$ & $\begin{array}{c}T_{\text {eff }}{ }^{\mathrm{K}} \\
\mathrm{K}\end{array}$ & $\begin{array}{c}\text { Cooling }^{\mathrm{b}} \\
\text { Age, Gyr }\end{array}$ & $\begin{array}{c}\text { Distance }^{\mathrm{c}} \\
\mathrm{pc}\end{array}$ & \multicolumn{1}{c}{$\begin{array}{c}v_{\text {tan }^{\mathrm{d}}} \\
\mathrm{km} \mathrm{s}^{-1}\end{array}$} \\
\hline ULAS J0121-00 & DC & unconstrained & $4480 \pm 230$ & $7.3_{-3.7}^{+2.0}$ & $81 \pm 19$ & $38 \pm 10[51]$ \\
ULAS J0826-00 & DC & $\mathrm{H}$ & $4170 \pm 130$ & $8.6_{-2.6}^{+1.3}$ & $76 \pm 10$ & $33 \pm 9[46]$ \\
ULAS J0840+05 & DC & mixed & $4350 \pm 110$ & $7.5_{-3.0}^{+0.4}$ & $82 \pm 15$ & $44 \pm 9[50]$ \\
ULAS J1006+09 & DC & He & $4260 \pm 100$ & $7.7_{-3.2}^{+0.4}$ & $73 \pm 17$ & $51 \pm 12[50]$ \\
ULAS J1206+03 & DC & He & $4610 \pm 80$ & $6.9_{-3.2}^{+0.8}$ & $94 \pm 20$ & $73 \pm 15[71]$ \\
SDSS J1247+06 & DQpec & mixed & 5120 & 5.6 & 60 & 110 \\
ULAS J1320+08 & DC & high-He mix & $4800 \pm 130$ & $6.5_{-3.0}^{+0.8}$ & $102 \pm 18$ & $95 \pm 19[101]$ \\
ULAS J1323+12 & DA & H & $5260 \pm 90$ & $4.3_{-2.1}^{+2.9}$ & $103 \pm 21$ & $163 \pm 30[102]^{\mathrm{f}}$ \\
ULAS J1345+15 & DC & $\mathrm{H}$ & $4120 \pm 150$ & $8.7_{-2.6}^{+1.4}$ & $58 \pm 8$ & $72 \pm 10[71]$ \\
ULAS J1351+12 & DC & He & $4810 \pm 70$ & $6.5_{-3.2}^{+0.9}$ & $103 \pm 21$ & $59 \pm 12[51]$ \\
ULAS J1404+13 & DC & mixed & $4380 \pm 100$ & $7.5_{-2.9}^{+0.4}$ & $59 \pm 10$ & $43 \pm 8[40]$ \\
ULAS J1436+05 & DC & unconstrained & $4340 \pm 170$ & $7.8_{-3.7}^{+2.0}$ & $57 \pm 12$ & $85 \pm 15[81]$ \\
ULAS J1454-01 & DC & He & $4800 \pm 60$ & $6.5_{-3.2}^{+0.9}$ & $65 \pm 15$ & $65 \pm 15[83]$ \\
ULAS J2331+15 & DA & H & $4970 \pm 80$ & $5.8_{-3.1}^{+3.7}$ & $95 \pm 27$ & $71 \pm 20[67]$ \\
\hline
\end{tabular}

a The uncertainty in $T_{\text {eff }}$ is due to both photometric scatter and an allowed range in gravity of $7.7 \leq \log g \leq 8.3$.

${ }^{\mathrm{b}}$ The cooling age is derived from the composition and temperature using the cooling models of Fontaine, Brassard \& Bergeron (2001). The uncertainty is due to the range in gravity of $7.7 \leq \log g \leq$ 8.3 .

${ }^{\mathrm{c}}$ The distance is estimated from the modelled and observed magnitudes (Holberg \& Bergeron 2006). The uncertainty is due to the range in gravity of $7.7 \leq \log g \leq 8.3$.

${ }^{\mathrm{d}}$ The tangential velocity is calculated from distance and the proper motion given in Table 1. Values in brackets are those implied by the alternative proper motion given in Table 1 .

ePreviously discovered in SDSS by Kilic et al. (2010a), tabulated properties are from that work.

${ }^{\mathrm{f}}$ The alternative value for $v_{\tan }$ is preferred, given the large uncertainty in the SDSS-LAS proper motion caused by the short timeline.

${ }^{\mathrm{g}}$ The fit to this white dwarf is poor (see Figure 11). 